\title{
Vanishing and comparison theorems in rigid analytic geometry
}

\author{
David Hansen*
}

February 25, 2019

\begin{abstract}
We prove a rigid analytic analogue of the Artin-Grothendieck vanishing theorem. Precisely, we prove (under mild hypotheses) that the geometric étale cohomology of any Zariskiconstructible sheaf on any affinoid rigid space $X$ vanishes in all degrees above the dimension of $X$. Along the way, we show that branched covers of normal rigid spaces can often be extended across closed analytic subsets, in analogy with a classical result for complex analytic spaces. We also prove some new comparison theorems relating the étale cohomology of schemes and rigid analytic varieties, and give some applications of them. In particular, we prove a structure theorem for Zariski-constructible sheaves on characteristic zero affinoid spaces.
\end{abstract}

\section{Contents}

1 Introduction $\quad \mathbf{2}$

1.1 Artin-Grothendieck vanishing . . . . . . . . . . . . . . . . 2

1.2 Comparison theorems and applications . . . . . . . . . . . . . 5

2 Preliminaries $\quad \mathbf{8}$

2.1 Zariski-constructible sheaves on rigid spaces . . . . . . . . . . . . 8

2.2 Extending covers across closed subsets . . . . . . . . . . . . . . . 10

3 Vanishing theorems $\quad 13$

3.1 The reduction step . . . . . . . . . . . . . . . . . . . . 13

3.2 Constant coefficients . . . . . . . . . . . . . . . . . . 15

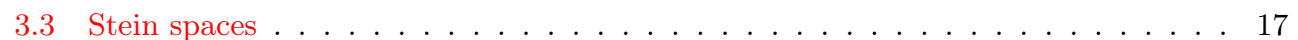

4 Comparison theorems $\quad 18$

4.1 The affinoid comparison theorem . . . . . . . . . . . . . . . . . 18

4.2 The relative comparison theorem . . . . . . . . . . . . . . . . . . . . . 19

4.3 Applications . . . . . . . . . . . . . . . . . . 23

References

25

*Department of Mathematics, Columbia University, 2990 Broadway, New York NY 10027; hansen@math.columbia.edu 


\section{Introduction}

This paper touches on several topics in the étale cohomology of non-archimedean analytic spaces. On the one hand, we prove an analogue of the Artin-Grothendieck vanishing theorem. ${ }^{1}$ This involves the class of so-called Zariski-constructible étale sheaves on rigid analytic spaces, which we define and study in this paper. On the other hand, as part of this study, we prove some new comparison theorems for étale cohomology, both for affinoid rings and for schemes over affinoid rings. Using these comparison results, we prove a strong structure theorem for Zariski-constructible sheaves on characteristic zero affinoid rigid spaces, and show that Zariski-constructibility is preserved under certain of the six functor operations.

\subsection{Artin-Grothendieck vanishing}

Let $X \subset \mathbf{C}^{m}$ be a smooth affine variety over $\mathbf{C}$, or more generally any complex Stein manifold. According to a classical theorem of Andreotti and Frankel [AF59], $X$ has the homotopy type of a CW complex of real dimension $\leq \operatorname{dim} X$. In particular, the cohomology groups $H^{i}(X, A)$ vanish for any abelian group $A$ and any $i>\operatorname{dim} X$. This vanishing theorem was significantly generalized by Artin and Grothendieck, who proved the following striking result.

Theorem 1.1 (Corollaire XIV.3.2 in [SGA73]). Let $X$ be an affine variety over a separably closed field $k$, and let $\mathscr{F}$ be any torsion abelian sheaf on the étale site of $X$. Then

$$
H_{\text {êt }}^{i}(X, \mathscr{F})=0
$$

for all $i>\operatorname{dim} X$.

We remind the reader that for a general $k$-variety $X$, the groups $H_{\text {ét }}^{i}(X, \mathscr{F})$ vanish in degrees $i>2 \operatorname{dim} X$, and this bound is sharp.

It's natural to wonder whether there is a rigid analytic analogue of the Artin-Grothendieck vanishing theorem. ${ }^{2}$ Again, we have a general sharp vanishing theorem due to Berkovich and Huber (cf. [Ber93, Corollary 4.2.6], [Hub96, Corollary 2.8.3]): for any quasicompact and quasiseparated ${ }^{3}$ rigid space $X$ over a complete algebraically closed nonarchimedean field $C$, and any torsion abelian sheaf $\mathscr{F}$ on $X_{\text {ét }}$, the cohomology group $H_{\text {êt }}^{i}(X, \mathscr{F})$ vanishes for all $i>2 \operatorname{dim} X$. Now in rigid geometry the affinoid spaces play the role of basic affine objects, and the most naive guess for an analogue of Artin-Grothendieck vanishing would be that $H_{\text {ét }}^{i}(X, \mathscr{F})$ vanishes for all affinoids $X / C$, all torsion abelian sheaves $\mathscr{F}$ on $X_{\text {ét }}$ and all $i>\operatorname{dim} X$.

Unfortunately, after some experimentation, one discovers that this fails miserably: there are plenty of torsion abelian sheaves on the étale site of any $d$-dimensional affinoid with nonzero cohomology in all degrees $i \in[0,2 d]$. For example, let $X / C$ be any (reduced) $d$-dimensional affinoid, and let $j: U \rightarrow X$ be the open inclusion of any small $d$-dimensional affinoid ball with $U$ strictly contained in $X$. Set $\mathscr{F}=j ! \mathbf{Z} / n \mathbf{Z}$ for some $n$ prime to the residue characteristic of $C$. Then $H_{\text {ét }}^{i}(X, \mathscr{F})=H_{c, \text { ét }}^{i}(U, \mathbf{Z} / n \mathbf{Z})$, and the latter group is Poincaré dual to $H_{\text {ét }}^{2 d-i}(U, \mathbf{Z} / n \mathbf{Z})$, which is nonzero when $i=2 d$. In particular, $H_{\text {êt }}^{2 d}(X, \mathscr{F}) \neq 0$.

\footnotetext{
${ }^{1}$ This result is often attributed to Artin alone. However, Artin suggested in a private communication that it should properly be attributed as joint work with Grothendieck.

${ }^{2}$ In this paper, we work with rigid analytic spaces and their étale cohomology using Huber's adic spaces [Hub96], but our results could easily be formulated using Berkovich spaces instead.

${ }^{3}$ (More generally, one can allow any quasiseparated rigid space admitting a covering by countably many quasicompact open subsets.)
} 
In this example, the sheaf $\mathscr{F}$ is constructible in a certain weak sense [Hub96, Def. 2.7.2], but it is far from the type of constructible étale sheaves one traditionally encounters in algebraic geometry, which are locally constant after passing to some locally closed strata in the Zariski topology. In particular, the formulation of the following conjecture rules out counterexamples like this, and seems to be a reasonable salvage of Artin-Grothendieck vanishing.

Conjecture 1.2. Let $X$ be an affinoid rigid space over a complete algebraically closed nonarchimedean field $C$, and let $\mathscr{G}$ be any Zariski-constructible sheaf of $\mathbf{Z} / n \mathbf{Z}$-modules on $X_{\text {ét }}$ for some $n$ prime to the residue characteristic of $C$. Then

$$
H_{\text {êt }}^{i}(X, \mathscr{G})=0
$$

for all $i>\operatorname{dim} X$.

Here for a given rigid space $X$ and Noetherian coefficient ring $\Lambda$, we say a sheaf $\mathscr{G}$ of $\Lambda$-modules on $X_{\text {ét }}$ is Zariski-constructible if $X$ admits a locally finite stratification into subspaces $Z_{i} \subset X$, each locally closed for the Zariski topology on $X$, such that $\left.\mathscr{G}\right|_{Z_{i, \text { et }}}$ is a locally constant sheaf of $\Lambda$-modules of finite type for each $i$. We denote the category of such sheaves by $\operatorname{Sh}_{z c}(X, \Lambda)$. We will see that $\operatorname{Sh}_{z c}(X, \Lambda)$ is a thick abelian subcategory of the category $\operatorname{Sh}(X, \Lambda)$ of all sheaves of $\Lambda$-modules on $X_{\text {ét }}$. In the derived setting, we say that a complex of étale sheaves $A \in D(X, \Lambda)$ is Zariski-constructible if it has Zariski-constructible cohomology sheaves; such objects form a saturated triangulated subcategory $D_{z c}(X, \Lambda)$. Note that Zariski-constructible sheaves are overconvergent, and so it is immaterial whether one interprets their étale cohomology in the framework of Berkovich spaces or adic spaces (cf. [Hub96, Theorem 8.3.5]).

The first main result of this paper confirms Conjecture 1.2 in the case where $C$ has characteristic zero and the pair $(X, \mathscr{G})$ arises via base extension from a discretely valued nonarchimedean field.

Theorem 1.3. Let $X$ be an affinoid rigid space over a complete discretely valued nonarchimedean field $K$ of characteristic zero, and let $\mathscr{F}$ be any Zariski-constructible sheaf of $\mathbf{Z} / n \mathbf{Z}$-modules on $X_{\text {ét }}$ for some $n$ prime to the residue characteristic of $K$. Then the cohomology groups $H_{\mathrm{et}}^{i}\left(X_{\widehat{K}}, \mathscr{F}\right)$ are finite for all $i$, and

$$
H_{\text {ét }}^{i}\left(X_{\widehat{K}}, \mathscr{F}\right)=0
$$

for all $i>\operatorname{dim} X$.

For a slightly more general result, see Corollary 3.4. As far as we know, this is the first progress on Conjecture 1.2 since Berkovich [Ber96] treated some cases where $\mathscr{F}=\mathbf{Z} / n \mathbf{Z}$ is constant and $X$ is assumed algebraizable in a certain sense. In particular, using a deep algebraization theorem of Elkik [Elk73, Théorème 7], Berkovich proved Conjecture 1.2 when $\mathscr{F}$ is constant and $X$ is smooth, which might give one some confidence in the general conjecture.

Our proof of Theorem 1.3 doesn't explicitly use any algebraization techniques. Instead, we reduce to the special case where $\mathscr{F}$ is constant. In this situation, it turns out we can argue directly, with fewer assumptions on $K$ :

Theorem 1.4. Let $X$ be an affinoid rigid space over a complete discretely valued nonarchimedean field $K$. Then

$$
H_{\text {ét }}^{i}\left(X_{\widehat{K}}, \mathbf{Z} / n \mathbf{Z}\right)=0
$$

for all $i>\operatorname{dim} X$ and all $n$ prime to the residue characteristic of $K$. 
The proof of this theorem uses a number of ingredients, including some theorems of Greco and Valabrega on excellent rings, a remarkable formula of Huber for the stalks of the nearby cycle sheaves $R^{q} \lambda_{*}(\mathbf{Z} / n \mathbf{Z})$ on $\operatorname{Spec}\left(A^{\circ} / \varpi\right)_{\text {et }}$, a special case of Gabber's delicate "affine Lefschetz theorem" for quasi-excellent schemes, and the classical Artin-Grothendieck vanishing theorem. We note that the proof of Gabber's theorem makes heavy use of algebraization techniques.

The reduction step involves an ingredient which seems interesting in its own right. To explain this, we make the following definition.

Definition 1.5. Let $X$ be a normal rigid space. A cover of $X$ is a finite surjective map $\pi: Y \rightarrow X$ from a normal rigid space $Y$, such that there exists some closed nowhere-dense analytic subset $Z \subset X$ with $\pi^{-1}(Z)$ nowhere-dense and such that $Y \backslash \pi^{-1}(Z) \rightarrow X \backslash Z$ is finite étale.

We then have the following result, which seems to be new.

Theorem 1.6. Let $X$ be a normal rigid space over a complete nonarchimedean field $K$, and let $Z \subset X$ be any closed nowhere-dense analytic subset. Then the restriction functor

$$
\begin{aligned}
\left\{\begin{array}{c}
\text { covers of } X \\
\text { étale over } X \backslash Z
\end{array}\right\} & \left.\rightarrow \begin{array}{c}
\text { finite étale covers } \\
\text { of } X \backslash Z
\end{array}\right\} \\
Y & \mapsto Y \times_{X}(X \backslash Z)
\end{aligned}
$$

is fully faithful. Moreover, if $K$ has characteristic zero, it is an equivalence of categories; in other words, any finite étale cover of $X \backslash Z$ extends uniquely to a cover of $X$.

We remind the reader that in the schemes setting, the analogue of the equivalence in Theorem 1.6 is an easy exercise in taking normalizations, and holds essentially whenever the base scheme $X$ is Nagata, while for complex analytic spaces the problem was solved by Stein and Grauert-Remmert in the 50's, cf. [DG94].

Let us say something about the proof. Full faithfulness is an easy consequence of Bartenwerfer's rigid analytic version of Riemann's Hebbarkeitssatz, which says that bounded functions on normal rigid spaces extend uniquely across nowhere-dense closed analytic subsets. Essential surjectivity in characteristic zero is more subtle; indeed, it provably fails in positive characteristic. When $X$ is smooth and $Z$ is a strict normal crossings divisor, however, essential surjectivity was proved by Lütkebohmert in his work [Lüt93] on Riemann's existence problem. ${ }^{4}$ We reduce the general case to Lütkebohmert's result using recent work of Temkin on embedded resolution of singularities for quasi-excellent schemes in characteristic zero.

We also note that our argument for the reduction of Theorem 1.3 to Theorem 1.4 reduces Conjecture 1.2 to the special case where $\mathscr{F}$ is constant, at least for $C$ of characteristic zero. With Theorem 1.4 in hand, this might put the general characteristic zero case of Conjecture 1.2 within reach of some approximation argument.

We also note that Conjecture 1.2 may well still hold true without restricting to $n$ coprime to the residue characteristic, but our evidence in this case is rather weaker, and $p$-adic étale cohomology of affinoids over $p$-adic fields behaves rather badly. As such, we have chosen not to explicitly formulate the conjecture in that generality.

\footnotetext{
${ }^{4}$ Although curiously, Lütkebohmert doesn't explicitly state the result in his paper, nor does he discuss full faithfulness.
} 


\subsection{Comparison theorems and applications}

The next main result in this paper is the following theorem, which gives some further justification for the definition of Zariski-constructible sheaves.

Theorem 1.7. Let $S=\operatorname{Spa} A$ be an affinoid rigid space over some characteristic zero nonarchimedean field $K$, and write $\mathcal{S}=\operatorname{Spec} A$, so there is a natural map of sites $\mu_{S}: S_{\text {ét }} \rightarrow \mathcal{S}_{\text {ét }}$. Let $\Lambda$ be a Noetherian coefficient ring killed by some positive integer, such that either $\# \Lambda$ is finite or the residue charactistic of $K$ is invertible in $\Lambda$. Let $\operatorname{Sh}_{c}(\mathcal{S}, \Lambda)$ denote the category of constructible sheaves of $\Lambda$-modules on $\mathcal{S}_{\text {ét }}$.

Then the functor

$$
\mu_{S}^{*}: \operatorname{Sh}_{c}(\mathcal{S}, \Lambda) \rightarrow \operatorname{Sh}_{z c}(S, \Lambda)
$$

is an equivalence of categories.

Note that we still expect full faithfulness in positive characteristic, but essential surjectivity definitely fails. For an explicit counterexample, take $K=\mathbf{F}_{p}((t))$ and $S=\operatorname{Spa} K\langle T\rangle$. Write $j: S^{\times}=S \backslash\{T=0\} \rightarrow S$ for the inclusion of the punctured disk. Then (for any $\Lambda$ ) there exists a locally constant constructible sheaf $\mathscr{F}$ on $S^{\times}$such that $j_{!} \mathscr{F}$ is not in the essential image of $\mu_{S}^{*}$ : precisely, one can choose an Artin-Schreier cover $\pi: U \rightarrow S^{\times}$defined by an equation $T^{p}-T-f$ for some well-chosen $f \in \mathcal{O}\left(S^{\times}\right)$, such that $\mathscr{F}=\pi_{*} \Lambda$ gives an example of the desired shape. The point is to choose an $f$ which has an especially bad essential singularity at $T=0$. This counterexample is closely related to the failure of Theorem 1.6 in positive characteristic.

Although intuitively reasonable, Theorem 1.7 is definitely not trivial to prove. The main tools in the proof are Theorem 1.6 together with two new comparison theorems for étale cohomology. To state the latter results, fix an affinoid $S=$ Spa $A$ over some nonarchimedean field $K$, and let $\mathcal{S}=\operatorname{Spec} A$ and $\mu_{S}$ be as above. Recall that for any locally finite type $\mathcal{S}$-scheme $\mathcal{X}$, there is a functorially associated rigid space $X=\mathcal{X}^{\text {an }}$ over $S$ together with a morphism $X \rightarrow \mathcal{X}$ of locally ringed spaces characterized by a simple universal property, and again there is a functorial map of sites $\mu_{X}: X_{\text {ét }} \rightarrow \mathcal{X}_{\text {ét }}$. We then have the following relative comparison theorem.

Theorem 1.8 (Relative comparison theorem). Assume that $\operatorname{char}(K)=0$, and let $f: \mathcal{X} \rightarrow \mathcal{Y}$ be a finite type morphism between $\mathcal{S}$-schemes locally of finite type, with analytification $f^{\text {an }}: X \rightarrow Y$. Let $\Lambda$ be a Noetherian coefficient ring killed by some positive integer, such that either \# $\Lambda$ is finite or the residue charactistic of $K$ is invertible in $\Lambda$. Then for any $F \in D_{c}^{+}(\mathcal{X}, \Lambda)$, the natural comparison map

$$
\mu_{Y}^{*} R f_{*} F \rightarrow R f_{*}^{\text {an }} \mu_{X}^{*} F
$$

is an isomorphism in $D(Y, \Lambda)$.

In the special cases where $f$ is proper or $\mathcal{S}=\operatorname{Spec} K$ is a point, this was proved by Berkovich and Huber in the 90s ([Ber93, Corollaries 7.1.4 and 7.5.4], [Hub96, Theorems 3.7.2 and 3.8.1]).

On the other hand, we also prove an "absolute" comparison theorem for affinoid adic spaces.

Theorem 1.9 (Affinoid comparison theorem). Let $\left(A, A^{+}\right)$be any strongly Noetherian Tate-Huber pair, with $S=\operatorname{Spa}\left(A, A^{+}\right)$the associated affinoid adic space. Write $\mathcal{S}=\operatorname{Spec} A$, so there is a map of sites $\mu_{S}: S_{\text {ét }} \rightarrow \mathcal{S}_{\text {ét }}$ as above. Then for any torsion abelian sheaf $\mathscr{F}$ on $\mathcal{S}_{\text {ét }}$, the natural comparison map

$$
H_{\text {ét }}^{n}(\mathcal{S}, \mathscr{F}) \rightarrow H_{\text {ét }}^{n}\left(S, \mu_{S}^{*} \mathscr{F}\right)
$$

is an isomorphism for all $n$. 
When $\mathscr{F}$ is a constant sheaf, this is proved in Huber's book, and one might hope to reduce to this case by a trick. This turns out to be surprisingly easy.

The proof of Theorem 1.8 is somewhat more involved. The rough idea is to reduce the general case by a series of dévissages to the very special case where $\mathcal{Y}$ is regular, $f$ is a dense open immersion, and $F=\underline{M}$ is a constant constructible sheaf of $\Lambda$-modules. This special case is handled by a completely straightforward adaptation of the final stages in the proof of [SGA73, Th. XVI.4.1], making crucial use of a cohomological purity theorem due to Huber; the latter theorem is where the conditions on $\Lambda$ in Theorem 1.8 arise. The reduction steps use a number of ingredients, including Temkin's results on resolution of singularities, Gabber's results on the étale cohomology of quasi-excellent schemes, and the truth of Theorem 1.8 for proper $f$. We note that Huber already suggested in his book that Theorem 1.8 should be true and provable along these lines, cf. [Hub96, p. 162].

Putting these comparison theorems together, we get the following very useful result.

Theorem 1.10. Let $\mathcal{S}=\operatorname{Spec} A$ be the spectrum of a characteristic zero affinoid, and let $f: \mathcal{X} \rightarrow \mathcal{S}$ be an $\mathcal{S}$-scheme of finite type with analytification $f^{\text {an }}: X \rightarrow S=\operatorname{Spa} A$. Let $\Lambda$ be a Noetherian coefficient ring killed by some positive integer, such that either $\# \Lambda$ is finite or the residue charactistic of $K$ is invertible in $\Lambda$. Then

i. For any $F \in D_{c}^{+}(\mathcal{X}, \Lambda)$, the natural map $R \Gamma(\mathcal{X}, F) \rightarrow R \Gamma\left(X, \mu_{X}^{*} F\right)$ is an isomorphism. Moreover, the natural adjunction map $F \rightarrow R \mu_{X *} \mu_{X}^{*} F$ is an isomorphism.

ii. The functor $\mu_{X}^{*}: D_{c}^{b}(\mathcal{X}, \Lambda) \rightarrow D(X, \Lambda)$ is fully faithful. More generally, there is a natural isomorphism

$$
\operatorname{Hom}_{D(X, \Lambda)}\left(\mu_{X}^{*} G, \mu_{X}^{*} F\right) \cong \operatorname{Hom}_{D(\mathcal{X}, \Lambda)}(G, F)
$$

for any $F \in D_{c}^{+}(\mathcal{X}, \Lambda)$ and $G \in D^{-}(\mathcal{X}, \Lambda)$.

Note that even if $\mathcal{X}=\mathcal{S}$, the proof of Theorem 1.10 requires the full power of the relative comparison theorem, since one needs to compare the étale cohomology of $\mathcal{U}$ and $\mathcal{U}^{\text {an }}$ for an arbitrary quasi-compact étale map $\mathcal{U} \rightarrow \mathcal{S}$, in which case $\mathcal{U}^{\text {an }}$ is typically never affinoid. This was our original motivation for proving the relative comparison theorem.

Finally, we take some first steps towards showing that Zariski-constructibility interacts well with the six operations in the étale cohomology of rigid spaces. We caution the reader that unless $f$ is proper, the functors $R f_{*}$ and $R f_{!}$typically destroy Zariski-constructibility, even if $f$ is the inclusion of a Zariski-open subset. ${ }^{5}$ However, we expect that this is more or less the only thing that goes wrong.

Theorem 1.11. Let $S$ be a characteristic zero affinoid space over a nonarchimedean field $K$, and set $\Lambda=\mathbf{Z} / n \mathbf{Z}$ for some $n$ prime to the residue characteristic of $K$. Let $g: S \rightarrow$ SpaK be the structure map, and let $\omega_{S}=R g ! \Lambda$ be the dualizing complex. Then

i. If $j: U \rightarrow S$ is the inclusion of a Zariski-open subspace and $\mathscr{F} \in \operatorname{Sh}(U, \Lambda)$ is locally constant constructible, then $R j_{*} \mathscr{F}$ is Zariski-constructible.

ii. If $\mathscr{F}, \mathscr{G} \in D^{-}(S, \Lambda)$ are Zariski-constructible, then so is $\mathscr{F} \otimes_{\Lambda}^{\mathbf{L}} \mathscr{G}$.

iii. The Verdier duality functor

$$
\mathbf{D}_{S}(-)=R \mathscr{H} \operatorname{om}_{S}\left(-, \omega_{S}\right)
$$

\footnotetext{
${ }^{5}$ For instance, let $S=\mathrm{Spa} \mathbf{Q}_{p}\langle T\rangle$, and set $S^{\times}=S \backslash\{T=0\}$ and $U=S^{\times} \backslash \cup_{n>1} V\left(T-p^{n}\right)$. Let $j: S^{\times} \rightarrow S$ and $h: U \rightarrow S^{\times}$denote the evident Zariski-open immersions. Then by design, $U$ is Zariski-open in $S^{\times}$but not in $S$ (in fact $S \backslash U$ is Zariski-dense in $S$ ), so $\mathscr{F}=h ! \Lambda$ is Zariski-constructible but $j ! \mathscr{F}$ is not.
} 
sends $D^{ \pm}(S, \Lambda)$ into $D^{\mp}(S, \Lambda)$, and if $\mathscr{F} \in D(S, \Lambda)$ is Zariski-constructible, then the Verdier dual $\mathbf{D}_{S} \mathscr{F}$ is Zariski-constructible as well. In particular, the dualizing complex $\omega_{S}$ is Zariskiconstructible. Moreover, if $\mathscr{F} \in D(S, \Lambda)$ is Zariski-constructible, then it is reflexive: the natural biduality map $\mathscr{F} \rightarrow \mathbf{D}_{S} \mathbf{D}_{S} \mathscr{F}$ is an isomorphism.

iv. If $\mathscr{F} \in D^{-}(S, \Lambda)$ and $\mathscr{G} \in D^{+}(S, \Lambda)$ are Zariski-constructible, then so is $R \mathscr{H} \operatorname{om}_{S}(\mathscr{F}, \mathscr{G})$.

v. If $f: T \rightarrow S$ is any map of affinoid rigid spaces and $\mathscr{F} \in D(S, \Lambda)$ is Zariski-constructible, then so is $R f^{!} \mathscr{F}$.

The irritating restriction to affinoid spaces here would disappear if one could settle the following fundamental problem.

Conjecture 1.12. Zariski-constructibility is an étale-local property. More precisely, if $f: Y \rightarrow X$ is any surjective étale map of rigid spaces and $\mathscr{F} \in \operatorname{Sh}(X, \Lambda)$ is any étale sheaf such that $f^{*} \mathscr{F}$ is Zariski-constructible, then $\mathscr{F}$ is Zariski-constructible.

We note that Huber has also defined a notion of Zariski-constructible sheaves on rigid spaces: a sheaf is Zariski-constructible in his sense if it is étale-locally Zariski-constructible in our sense. In particular, Conjecture 1.12 implies that Huber's definition coincides with ours. Conjecture 1.12 also implies (upon combining it with Theorem 1.7) that sheaves which are Zariski-constructible in our sense coincide with the constructible sheaves defined by Berkovich in [Ber15, §1], at least in characteristic zero.

Conjecture 1.12 can be deduced from the following conjecture, which looks very plausible.

Conjecture 1.13. Let $\mathscr{F} \in \operatorname{Sh}(X, \Lambda)$ be a Zariski-constructible sheaf on an affinoid rigid space $X$, and let $\mathcal{U}_{X}(\mathscr{F})$ denote the largest open subset $U \subset X$ such that $\mathscr{F} \mid U$ is locally constant. Then $\mathcal{U}_{X}(\mathscr{F}) \subset X$ is Zariski-open.

We end this introduction with one more tantalizing problem, which seems to really require a new idea.

Conjecture 1.14. Let $f: X \rightarrow Y$ be a proper morphism of rigid spaces. If $F \in D^{+}(X, \Lambda)$ is Zariski-constructible, then so is $R f_{*} F$.

\section{Remarks on terminology and conventions.}

Our convention is that a "nonarchimedean field" is a topological field whose topology is defined by a nontrivial nonarchimedean valuation of rank one. If $K$ is any nonarchimedean field, we regard rigid analytic spaces over $K$ as a full subcategory of the category of adic spaces locally of topologically finite type over $\operatorname{Spa}\left(K, K^{\circ}\right)$. If $A$ is any topological ring, we write $A^{\circ}$ for the subset of powerbounded elements; if $A$ is a Huber ring, we write $\operatorname{Spa} A$ for $\operatorname{Spa}\left(A, A^{\circ}\right)$. Unless explicitly stated otherwise, all sheaves are étale sheaves, all derived categories are derived categories of étale sheaves, all cohomology groups are étale cohomology groups, etc.

We use the terms "Zariski-closed subset" and "closed analytic subset" interchangeably, and we always regard Zariski-closed subsets of rigid spaces as rigid spaces via the induced reduced structure. Finally, we remind the reader that in rigid geometry the phrases "dense Zariski-open subset" and "Zariski-dense open subset" have very different meanings. 


\section{Acknowledgments}

Several years ago, Giovanni Rosso and John Welliaveetil asked me whether anything was known about the $l$-cohomological dimension of affinoid rigid spaces, and I'd like to thank them very heartily for this crucial initial stimulation.

Johan de Jong listened to some of my early ideas about these problems and pointed me to the "Travaux de Gabber" volumes in response to my desperate search for tools. Christian Johansson planted in my head the usefulness of the excellence property for convergent power series rings. Peter Scholze read a rough initial draft and pointed out that certain arguments worked without change in equal characteristic zero; Scholze also inadvertently inspired a crucial trick (cf. footnote 9 ). Brian Conrad offered several helpful comments and corrections on a later draft. The anonymous referee gave some perceptive suggestions for clarifications and improvements. To all of these mathematicians, I'm very grateful.

\section{Preliminaries}

\subsection{Zariski-constructible sheaves on rigid spaces}

In this section we discuss some basics on Zariski-constructible étale sheaves on rigid spaces. For simplicity we fix a nonachimedean field $K$ and a Noetherian coefficient ring $\Lambda$; until further notice, roman letters $X, Y, \ldots$ denote rigid spaces over $K$, and "Zariski-constructible" means a Zariskiconstructible sheaf of $\Lambda$-modules, as defined in the introduction, on the étale site of some rigid space $X$ over $K$.

Proposition 2.1. Let $\mathscr{F}$ be a Zariski-constructible sheaf on a rigid space $X$.

i. If $f: Y \rightarrow X$ is any morphism of rigid spaces, then $f^{*} \mathscr{F}$ is Zariski-constructible.

ii. If $i: X \rightarrow W$ is a closed immersion, then $i_{*} \mathscr{F}$ is Zariski-constructible.

iii. If $j: X \rightarrow V$ is a Zariski-open immersion and $\mathscr{F}$ is locally constant, then $j ! \mathscr{F}$ is Zariskiconstructible.

Proof. Trivial.

We will often verify Zariski-constructibility via the following dévissage, which is a trivial consequence of the previous proposition.

Proposition 2.2. Let $X$ be any rigid space, and let $\mathscr{F}$ be a sheaf of $\Lambda$-modules on $X_{\text {ét }}$. The following are equivalent:

i. $\mathscr{F}$ is Zariski-constructible.

ii. There is some dense Zariski-open subset $j: U \rightarrow X$ with closed complement $i: Z \rightarrow X$ such that $i^{*} \mathscr{F}$ is Zariski-constructible and $j^{*} \mathscr{F}$ is locally constant of finite type.

Note that one cannot weaken the hypotheses in ii. here to the condition that $j^{*} \mathscr{F}$ is Zariskiconstructible; this is related to the fact that the Zariski topology in the rigid analytic world is not transitive.

Proposition 2.3. If $f: X^{\prime} \rightarrow X$ is a finite morphism and $\mathscr{F}$ is a Zariski-constructible sheaf on $X^{\prime}$, then $f_{*} \mathscr{F}$ is Zariski-constructible.

We note in passing that if $f: X \rightarrow Y$ is a finite morphism, or more generally any quasi-compact separated morphism with finite fibers, then $f_{*}: \operatorname{Sh}\left(X_{\text {ét }}, \Lambda\right) \rightarrow \operatorname{Sh}\left(Y_{\text {ét }}, \Lambda\right)$ is an exact functor, cf. Proposition 2.6.4 and Lemma 1.5.2 in [Hub96]. 
Proof. We treat the case where $X^{\prime}=\operatorname{Spa} A^{\prime}$ and $X=$ Spa $A$ are affinoid, which is all we'll need later. We can assume they are reduced and that $f$ is surjective. If $i: Z \rightarrow X$ is Zariski-closed and nowhere dense, then $\operatorname{dim} Z<\operatorname{dim} X$; setting $Z^{\prime}=Z \times_{X} X^{\prime}$ and writing $f^{\prime}: Z^{\prime} \rightarrow Z$ and $i^{\prime}: Z^{\prime} \rightarrow X^{\prime}$ for the evident morphisms, we can assume that $i^{*} f_{*} \mathscr{F} \cong f_{*}^{\prime} i^{\prime *} \mathscr{F}$ is Zariski-constructible by induction on $\operatorname{dim} X$. By dévissage, it now suffices to find a dense Zariski-open subset $j: U \rightarrow X$ such that $j^{*} f_{*} \mathscr{F}$ is locally constant. To do this, choose a dense Zariski-open subset $V \subset X^{\prime}$ such that $\left.\mathscr{F}\right|_{V}$ is locally constant. Then $W=X \backslash f\left(X^{\prime} \backslash V\right)$ is a dense Zariski-open subset of $X$, and $\mathscr{F}$ is locally constant after pullback along the open immersion $W^{\prime}=W \times_{X} X^{\prime} \rightarrow X^{\prime}$. If $\operatorname{char}(K)=0$, we now conclude by taking $U$ to be any dense Zariski-open subset contained in $W$ such that $U^{\prime}=U \times_{X} X^{\prime} \rightarrow U$ is finite étale; if $\operatorname{char}(K)=p$, we instead choose $U$ so that $U^{\prime} \rightarrow U$ factors as the composition of a universal homeomorphism followed by a finite étale map. (For the existence of such a $U$, look at the map of schemes Spec $A^{\prime} \rightarrow \operatorname{Spec} A$; this morphism has the desired structure over all generic points of the target, and these structures then spread out over a dense Zariski-open subset of Spec $A$. One then concludes by analytifying.)

Proposition 2.4. Let $X$ be a rigid space.

i. Let $0 \rightarrow \mathscr{F} \rightarrow \mathscr{G} \rightarrow \mathscr{H} \rightarrow 0$ be a short exact sequence of étale sheaves of $\Lambda$-modules on $X$. If two of the three sheaves $\{\mathscr{F}, \mathscr{G}, \mathscr{H}\}$ are Zariski-constructible, then so is the third.

ii. Let $f: \mathscr{F} \rightarrow \mathscr{G}$ be any map of Zariski-constructible sheaves. Then $\operatorname{ker} f, \operatorname{im} f$ and $\operatorname{coker} f$ are Zariski-constructible.

iii. Let $F \rightarrow G \rightarrow H \stackrel{[1]}{\rightarrow}$ be a distinguished triangle in $D(X, \Lambda)$. If two of the three objects $F, G, H$ are Zariski-constructible, then so is the third.

We often refer to parts i. and iii. as the "two-out-of-three property".

Proof. For i., by induction on $\operatorname{dim} X$ and dévissage, it suffices to find some dense Zariski-open subset $j: U \rightarrow X$ such that all three sheaves are locally constant after restriction to $U$. By assumption, we can choose $U$ such that two of the three sheaves have this property. Looking at the exact sequence $\left.\left.\left.0 \rightarrow \mathscr{F}\right|_{U} \rightarrow \mathscr{G}\right|_{U} \rightarrow \mathscr{H}\right|_{U} \rightarrow 0$, [Hub96, Lemma 2.7.3] implies that all three sheaves are constructible (in the sense of [Hub96]). By [Hub96, Lemma 2.7.11], it now suffices to check that all three sheaves are overconvergent. But if $\bar{x} \rightsquigarrow \bar{y}$ is any specialization of geometric points, this follows immediately by applying the snake lemma to the diagram

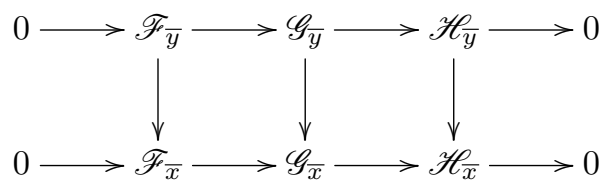

since by assumption two of the three vertical arrows are isomorphisms.

For ii., we first show that coker $f$ is Zariski-constructible. As in the argument for i., it suffices to find some dense Zariski-open subset $j: U \rightarrow X$ such that coker $\left.f\right|_{U}$ is locally constant. Choose $U$ such that $\left.\mathscr{F}\right|_{U}$ and $\left.\mathscr{G}\right|_{U}$ are locally constant, so coker $\left.f\right|_{U}$ is constructible. Then for $\bar{x} \rightsquigarrow \bar{y}$ any specialization of geometric points of $U$, the first two vertical maps in the diagram

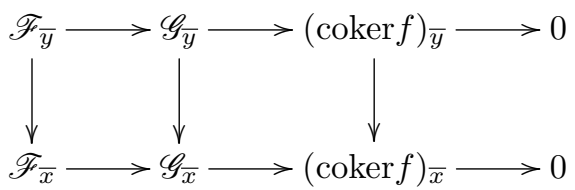


are isomorphisms by assumption, so the map $(\operatorname{coker} f)_{\bar{y}} \rightarrow(\operatorname{coker} f)_{\bar{x}}$ is an isomorphism by the five lemma. Thus coker $\left.f\right|_{U}$ is overconvergent and constructible, hence locally constant by [Hub96, Lemma 2.7.11].

The remaining parts of ii. now follow from the exact sequences $0 \rightarrow \operatorname{im} f \rightarrow \mathscr{G} \rightarrow \operatorname{coker} f \rightarrow 0$ and $0 \rightarrow \operatorname{ker} f \rightarrow \mathscr{F} \rightarrow \operatorname{im} f \rightarrow 0$ by successive applications of i.

For iii., we can assume (possibly after a shift) that $F$ and $H$ are Zariski-constructible. The result then follows from parts i. and ii., by looking at the exact sequences

$$
0 \rightarrow \operatorname{coker}\left(\mathscr{H}^{n-1}(H) \rightarrow \mathscr{H}^{n}(F)\right) \rightarrow \mathscr{H}^{n}(G) \rightarrow \operatorname{ker}\left(\mathscr{H}^{n}(H) \rightarrow \mathscr{H}^{n+1}(F)\right) \rightarrow 0 .
$$

Corollary 2.5. For any rigid space $X, \operatorname{Sh}_{z c}(X, \Lambda)$ is a thick abelian subcategory of $\operatorname{Sh}(X, \Lambda)$.

\subsection{Extending covers across closed subsets}

In this section we prove a slight strengthening of Theorem 1.6. We'll freely use basic facts about irreducible components of rigid spaces, as developed in [Con99], without any comment. The following result of Bartenwerfer [Bar76, §3] is also crucial for our purposes.

Theorem 2.6 (Bartenwerfer). Let $X$ be a normal rigid space, and let $Z \subset X$ be a nowheredense closed analytic subset, with $j: X \backslash Z \rightarrow X$ the inclusion of the open complement. Then $\mathcal{O}_{X}^{+} \stackrel{\sim}{\rightarrow} j_{*} \mathcal{O}_{X \backslash Z}^{+}$and $\mathcal{O}_{X} \stackrel{\sim}{\rightarrow}\left(j_{*} \mathcal{O}_{X \backslash Z}^{+}\right)\left[\frac{1}{\varpi}\right]$. In particular, if $X$ is affinoid and $f \in \mathcal{O}_{X}(X \backslash Z)$ is bounded, then $f$ extends uniquely to an element of $\mathcal{O}_{X}(X)$, so $\mathcal{O}_{X}(X) \cong \mathcal{O}_{X}^{+}(X \backslash Z)\left[\frac{1}{\varpi}\right]$.

Corollary 2.7. If $X$ is a connected normal rigid space and $Z \subset X$ is a nowhere-dense closed analytic subset, then $X \backslash Z$ is connected.

Proof. Any idempotent in $\mathcal{O}_{X}(X \backslash Z)$ is power-bounded, so this is immediate from the previous theorem.

Proposition 2.8. Let $X$ be a normal rigid space, and let $\pi: Y \rightarrow X$ be a cover of $X$. Then each irreducible component of $Y$ maps surjectively onto some irreducible component of $X$. Moreover, if $V \subset X$ is any closed nowhere-dense analytic subset, then $\pi^{-1}(V)$ is nowhere-dense.

Proof. We immediately reduce to the case where $X$ is connected. Let $Z \subset X$ be as in the definition of a cover, and let $Y_{i}$ be any connected component of $Y$, so then $Y_{i} \cap \pi^{-1}(Z)$ is closed and nowheredense in $Y_{i}$ and $Y_{i} \backslash Y_{i} \cap \pi^{-1}(Z) \rightarrow X \backslash Z$ is finite étale. Then

$$
\operatorname{im}\left(Y_{i} \backslash Y_{i} \cap \pi^{-1}(Z) \rightarrow X \backslash Z\right)
$$

is a nonempty open and closed subset of $X \backslash Z$, so it coincides with $X \backslash Z$ by the previous corollary. In particular, $\pi\left(Y_{i}\right)$ contains a dense subset of $X$. On the other hand, $\pi\left(Y_{i}\right)$ is a closed analytic subset of $X$ since $\pi$ is finite. Therefore $\pi\left(Y_{i}\right)=X$.

For the second claim, note that if $V$ is a closed analytic subset of a connected normal space $X$, then $V \subsetneq X$ if and only if $V$ is nowhere-dense if and only if $\operatorname{dim} V<\operatorname{dim} X$. Since

$$
\operatorname{dim} \pi^{-1}(V) \cap Y_{i}=\operatorname{dim} V<\operatorname{dim} X=\operatorname{dim} Y_{i}
$$

for any irreducible component $Y_{i}$ of $Y$, this gives the claim. 
Proposition 2.9. Let $X$ be a normal rigid space, and let $Z \subset X$ be any closed nowhere-dense analytic subset. Then the restriction functor

$$
\begin{aligned}
\{\text { covers of } X\} & \rightarrow\{\text { covers of } X \backslash Z\} \\
Y & \mapsto Y \times_{X}(X \backslash Z)
\end{aligned}
$$

is fully faithful.

Proof. If $\pi: Y \rightarrow X$ is any cover and $U \subset X$ is any open affinoid, then $\pi^{-1}(U)$ is affinoid as well, and $\pi^{-1}(Z \cap U)$ is nowhere-dense in $U$ by the previous proposition. But then $\mathcal{O}_{Y}\left(\pi^{-1}(U)\right) \cong$ $\mathcal{O}_{Y}^{+}\left(\pi^{-1}(U \backslash U \cap Z)\right)\left[\frac{1}{\varpi}\right]$ by Theorem 2.6, so $\mathcal{O}_{Y}(U)$ only depends on $Y \times_{X}(X \backslash Z)$. This immediately gives the result.

It remains to prove the following result.

Theorem 2.10. Let $X$ be a normal rigid space over a characteristic zero complete nonarchimedean field $K$, and let $Z \subset X$ be any closed nowhere-dense analytic subset. Then the restriction functor

$$
\begin{aligned}
\left\{\begin{array}{c}
\text { covers of } X \\
\text { étale over } X \backslash Z\}
\end{array}\right. & \rightarrow\left\{\begin{array}{c}
\text { finite étale covers } \\
\text { of } X \backslash Z
\end{array}\right\} \\
Y & \mapsto Y \times_{X}(X \backslash Z)
\end{aligned}
$$

is essentially surjective.

In other words, given a (surjective) finite étale cover $\pi: Y \rightarrow X \backslash Z$, we need to find a cover $\tilde{\pi}: \tilde{Y} \rightarrow X$ and an open immersion $Y \rightarrow \tilde{Y}$ such that the diagram

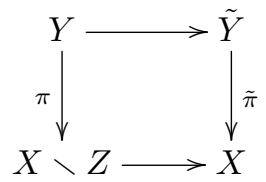

is cartesian. We refer to this as the problem of extending $Y$ to a cover of $X$. Note that by the full faithfulness proved above, we're always free to work locally on $X$ when extending a given cover of $X \backslash Z$.

Until further notice, fix $K$ of characteristic zero. The key special case is the following result.

Theorem 2.11 (Lütkebohmert). If $X$ is a smooth rigid space and $D \subset X$ is a strict normal crossings divisor, then any finite étale cover of $X \backslash D$ extends to a cover of $X$.

This is more or less an immediate consequence of the arguments in $\S 3$ in Lütkebohmert's paper [Lüt93] (and is implicit in the proof of Theorem 3.1 of loc. cit.). For the convenience of the reader, we explain the deduction in detail. Let $\mathbf{B}^{r}=$ Spa $K\left\langle X_{1}, \ldots, X_{r}\right\rangle$ denotes the $r$-dimensional closed affinoid ball.

Lemma 2.12 (Lemma 3.3 in [Lüt93]). Let $S$ be a smooth $K$-affinoid space, and let $r \geq 1$ be any integer. If $Y_{0}$ is a cover of $S \times\left(\mathbf{B}^{r} \backslash V\left(X_{1}, \ldots, X_{r}\right)\right)$ which is étale over $S \times\left(\mathbf{B}^{r} \backslash V\left(X_{1} \cdots X_{r}\right)\right)$, then $Y_{0}$ extends to a cover $\tilde{Y}$ of $S \times \mathbf{B}^{r}$.

We also need a result of Kiehl on the existence of "tubular neighborhoods" of strict normal crossings divisors in smooth rigid spaces. 
Lemma 2.13. If $D \subset X$ is a strict normal crossings divisor in a smooth rigid space, then for any (adic) point $x$ in $X$ contained in exactly $r$ irreducible components $D_{1}, \ldots, D_{r}$ of $D$, we can find some small open affinoid $U \subset X$ containing $x$ together with a smooth affinoid $S$ and an isomorphism $U \simeq S \times \mathbf{B}^{r}$, under which the individual components $D_{i} \cap U$ containing $x$ identify with the zero loci of the coordinate functions $X_{i} \in \mathcal{O}\left(\mathbf{B}^{r}\right)$.

Proof. This follows from a careful reading of Theorem 1.18 in [Kie67b] (cf. also [Mit, Theorem 2.11]).

Granted these results, we deduce Theorem 2.11 as follows. By full faithfulness we can assume that $X$ is quasicompact, or even affinoid. We now argue by induction on the maximal number $\iota(D)$ of irreducible components of $D$ passing through any individual point of $X$. If $\iota(D)=1$, then $D$ is smooth, so arguing locally around any point in $D$, Lemma 2.13 puts us exactly in the situation covered by the case $r=1$ of Lemma 2.12. If $\iota(D)=n$, then locally on $X$ we can assume that $D$ has (at most) $n$ smooth components $D_{1}, D_{2}, \ldots, D_{n}$. By the induction hypothesis, any finite étale cover $Y$ of $X \backslash D$ extends to a cover $Y_{i}$ of $X \backslash D_{i}$ for each $1 \leq i \leq n$, since $\iota\left(D \backslash D_{i}\right) \leq n-1$ for $D \backslash D_{i}$ viewed as a strict normal crossings divisor in $X \backslash D_{i}$. By full faithfulness the $Y_{i}$ 's glue to a cover $Y_{0}$ of $X \backslash \cap_{1 \leq i \leq n} D_{i}$, and locally around any point in $\cap_{1 \leq i \leq n} D_{i}$ Lemma 2.13 again puts us in the situation handled by Lemma 2.12, so $Y_{0}$ extends to a cover $\tilde{Y}$ of $X$, as desired.

Proof of Theorem 2.10. We can assume that $X=\operatorname{Spa} A$ is an affinoid rigid space, so $Z=\operatorname{Spa} B$ is also affinoid, and we get a corresponding closed immersion of schemes $\mathcal{Z}=\operatorname{Spec} B \rightarrow \mathcal{X}=\operatorname{Spec} A$. These are quasi-excellent schemes over $\mathbf{Q}$, so according to Theorem 1.11 in [Tem17], we can find a projective birational morphism $f: \mathcal{X}^{\prime} \rightarrow \mathcal{X}$ such that $\mathcal{X}^{\prime}$ is regular and $f^{-1}(\mathcal{Z})^{\text {red }}$ is a strict normal crossings divisor, and such that $f$ is an isomorphism away from $\mathcal{Z} \cup \mathcal{X}^{\text {sing }}$. Analytifying, we get a proper morphism of rigid spaces $g: X^{\prime} \rightarrow X$ with $X^{\prime}$ smooth such that $g^{-1}(Z)^{\text {red }}$ is a strict normal crossings divisor.

Suppose now that we're given a finite étale cover $Y \rightarrow X \backslash Z$. Base changing along $g$, we get a finite étale cover of $X^{\prime} \backslash g^{-1}(Z)$, which then extends to a cover $h: Y^{\prime} \rightarrow X^{\prime}$ by Theorem 2.11. Now, since $g \circ h$ is proper, the sheaf $(g \circ h)_{*} \mathcal{O}_{Y^{\prime}}$ defines a sheaf of coherent $\mathcal{O}_{X}$-algebras by [Kie67a]. Taking the normalization of the affinoid space associated with the global sections of this sheaf, we get a normal affinoid $Y^{\prime \prime}$ together with a finite map $Y^{\prime \prime} \rightarrow X$ and a canonical isomorphism $\left.\left.Y^{\prime \prime}\right|_{(X \backslash Z)^{\mathrm{sm}}} \cong Y\right|_{(X \backslash Z)^{\mathrm{sm}}}$. The cover $\tilde{Y} \rightarrow X$ we seek can then be defined as the Zariski closure of $\left.Y^{\prime \prime}\right|_{(X \backslash Z)^{\mathrm{sm}}}$ in $Y^{\prime \prime}$; note that this is just a union of irreducible components of $Y^{\prime \prime}$, so it's still normal, and it's easy to check that $\tilde{Y}$ is a cover of $X$. Finally, since $\tilde{Y}$ and $Y$ are canonically isomorphic after restriction to $(X \backslash Z)^{\mathrm{sm}}$, the full faithfulness argument shows that this isomorphism extends to an isomorphism $\left.\tilde{Y}\right|_{X \backslash Z} \cong Y$, since $(X \backslash Z)^{\mathrm{sm}}$ is a dense Zariski-open subset of $X \backslash Z$. This concludes the proof.

For completeness, we state the following mild generalization of Theorem 2.10.

Theorem 2.14. Let $X$ be a normal rigid space over a characteristic zero complete nonarchimedean field $K$, and let $V \subset X$ be any closed nowhere-dense analytic subset. Suppose that $Y \rightarrow X \backslash V$ is a cover, and that there is some closed nowhere-dense analytic set $W \subset X \backslash V$ such that $V \cup W$ is an analytic set in $X$ and such that

$$
Y \times_{(X \backslash V)}(X \backslash V \cup W) \rightarrow X \backslash V \cup W
$$

is finite étale. Then $Y$ extends to a cover $\tilde{Y} \rightarrow X$. 
Proof. Apply Theorem 2.10 with $Z=V \cup W$ to construct $\tilde{Y} \rightarrow X$ extending

$$
Y \times_{(X \backslash V)}(X \backslash V \cup W) \rightarrow X \backslash V \cup W,
$$

and then use full faithfulness to deduce that $\left.\tilde{Y}\right|_{X \backslash V} \cong Y$.

Combining this extension theorem with classical Zariski-Nagata purity, we get a purity theorem for rigid analytic spaces.

Corollary 2.15. Let $X$ be a smooth rigid analytic space over a characteristic zero complete nonarchimedean field, and let $Z \subset X$ be any closed analytic subset which is everywhere of codimension $\geq 2$. Then finite étale covers of $X$ are equivalent to finite étale covers of $X \backslash Z$.

We also get the following equivalence, which plays an important role in the proof of Theorem 1.7 .

Corollary 2.16. Let $S=\operatorname{Spa} A$ be a characteristic zero affinoid. Set $\mathcal{S}=\operatorname{Spec} A$, and let $\mathcal{U} \subset \mathcal{S}$

be any normal dense open subscheme, with $U \subset S$ the corresponding dense Zariski-open subspace of $S$. Then analytification induces an equivalence of categories $\mathcal{U}_{\text {fét }} \cong U_{\text {fét }}$.

Note that $\mathcal{U}$ is normal if and only if $U$ is normal; this follows from [BGR84, Proposition 7.3.2/8.i].

Proof. The essential point is to construct the functor $U_{\text {fét }} \mapsto \mathcal{U}_{\text {fét. }}$. For this, let $Y \rightarrow U$ be any finite étale cover. Letting $S^{\nu}$ and $\mathcal{S}^{\nu}$ denote the normalizations of $S$ and $\mathcal{S}$, the map $U \rightarrow S$ factors canonically over a dense Zariski-open immersion $U \rightarrow S^{\nu}$, and similarly for $\mathcal{U}$. By Theorem 2.10, $Y \rightarrow U$ extends to a cover $\tilde{Y} \rightarrow S^{\nu}$. Since $\tilde{Y} \rightarrow S^{\nu}$ is finite, $\tilde{Y}=\mathrm{Spa} B$ for $B$ some module-finite $A^{\nu}$-algebra. Then $\mathcal{Y}=\operatorname{Spec} B \times \mathcal{S}^{\nu} \mathcal{U} \rightarrow \mathcal{U}$ is the desired algebraization of $Y \rightarrow U$.

\section{Vanishing theorems}

\subsection{The reduction step}

In this section we deduce Theorem 1.3 from Theorem 1.4. For clarity we focus on the vanishing statement in the theorem; it's easy to see that the following argument also reduces the finiteness of the groups $H_{\text {êt }}^{i}\left(X_{\widehat{K}}, \mathscr{F}\right)$ to finiteness in the special case where $\mathscr{F}=\mathbf{Z} / n \mathbf{Z}$ is constant, and finiteness in the latter case follows from [Ber15, Theorem 1.1.1].

Proof of Theorem 1.3. Fix a nonarchimedean field $K$ and a coefficient ring $\Lambda=\mathbf{Z} / n \mathbf{Z}$ as in the theorem. In what follows, "sheaf" is shorthand for "étale sheaf of $\Lambda$-modules". For nonnegative integers $d, i$, consider the following statement.

Statement $\mathcal{T}_{d, i}$ : "For all $K$-affinoids $X$ of dimension $\leq d$, all Zariski-constructible sheaves $\mathscr{F}$ on $X$, and all integers $j>i$, we have $H_{\text {ét }}^{j}\left(X_{\widehat{K}}, \mathscr{F}\right)=0 . "$

We are trying to prove that $\mathcal{T}_{d, d}$ is true for all $d \geq 0$. The idea is to argue by ascending induction on $d$ and descending induction on $i$. More precisely, it clearly suffices to assume the truth of $\mathcal{T}_{d-1, d-1}$ and then show that $\mathcal{T}_{d, i+1}$ implies $\mathcal{T}_{d, i}$ for any $i \geq d$; as noted in the introduction, $\mathcal{T}_{d, 2 d}$ is true for any $d \geq 0$, which gives a starting place for the descending induction.

We break the details into several steps.

Step One. Suppose that $\mathcal{T}_{d-1, d-1}$ holds. Then for any d-dimensional affinoid $X$, any Zariskiconstructible sheaf $\mathscr{F}$ on $X$, and any dense Zariski-open subset $j: U \rightarrow X$, the natural map $H_{\text {êt }}^{i}\left(X_{\widehat{K}}, j_{!} j^{*} \mathscr{F}\right) \rightarrow H_{\text {êt }}^{i}\left(X_{\widehat{K}}, \mathscr{F}\right)$ is surjective for $i=d$ and bijective for $i>d$. 
Letting $i: Z \rightarrow X$ denote the closed complement, this is immediate by looking at the long exact sequence

$$
\cdots \rightarrow H_{\text {ét }}^{i-1}\left(Z_{\widehat{\bar{K}}}, i^{*} \mathscr{F}\right) \rightarrow H_{\text {ét }}^{i}\left(X_{\widehat{K}}, j ! j^{*} \mathscr{F}\right) \rightarrow H_{\text {ét }}^{i}\left(X_{\widehat{K}}, \mathscr{F}\right) \rightarrow H_{\text {ét }}^{i}\left(Z_{\widehat{\bar{K}}}, i^{*} \mathscr{F}\right) \rightarrow \cdots
$$

associated with the short exact sequence

$$
0 \rightarrow j ! j^{*} \mathscr{F} \rightarrow \mathscr{F} \rightarrow i_{*} i^{*} \mathscr{F} \rightarrow 0
$$

and then applying $\mathcal{T}_{d-1, d-1}$ to control the outer terms.

Step Two. For any $d, i, \mathcal{T}_{d, i}$ holds if and only if it holds for all normal affinoids.

One direction is trivial. For the other direction, note that by Noether normalization for affinoids [BGR84, Corollary 6.1.2/2], any $d$-dimensional affinoid $X$ admits a finite map

$$
\tau: X \rightarrow \mathbf{B}^{d}=\operatorname{Spa} K\left\langle T_{1}, \ldots, T_{d}\right\rangle,
$$

and $\tau_{*}=R \tau_{*}$ preserves Zariski-constructibility by Proposition 2.3.

Step Three. ${ }^{6}$ Suppose that $\mathcal{T}_{d-1, d-1}$ holds. Then for any d-dimensional normal affinoid $X$, any dense Zariski-open subset $j: U \rightarrow X$, and any locally constant constructible sheaf $\mathscr{H}$ on $U$, we can find a Zariski-constrictible sheaf $\mathscr{G}$ on $X$ together with a surjection $s: \mathscr{G} \rightarrow j ! \mathscr{H}$, such that moreover $H_{\text {ét }}^{i}\left(X_{\widehat{K}}, \mathscr{G}\right)=0$ for all $i>d$.

To prove this, suppose we are given $X, U$, and $\mathscr{H}$ as in the statement. By definition, we can find a finite étale cover $\pi: Y \rightarrow U$ such that $\pi^{*} \mathscr{H}$ is constant, i.e. such that there exists a surjection $\Lambda_{Y}^{n} \rightarrow \pi^{*} \mathscr{H}$ for some $n$; fix such a surjection. This is adjoint to a surjection $\pi_{!}\left(\Lambda_{Y}^{n}\right) \rightarrow \mathscr{H},{ }^{7}$ which extends by zero to a surjection $s: j ! \pi_{!}\left(\Lambda_{Y}^{n}\right) \rightarrow j ! \mathscr{H}$. We claim that the sheaf $\mathscr{G}=j ! \pi_{!}\left(\Lambda_{Y}^{n}\right)$ has the required properties. Zariski-constructibility is clear from the identification $\pi_{!}=\pi_{*}$ and Proposition 2.3. For the vanishing statement, we apply Theorem 1.6 to extend $Y \rightarrow U$ to a cover $\tilde{\pi}: \tilde{Y} \rightarrow X$ sitting in a cartesian diagram

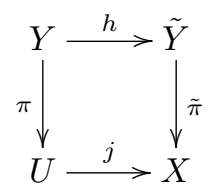

where $\tilde{Y}$ is a normal affinoid and $h$ is a dense Zariski-open immersion. By proper base change and the finiteness of $\tilde{\pi}$, we get isomorphisms

$$
\mathscr{G}=j_{!} \pi_{!}\left(\Lambda_{Y}^{n}\right) \cong \tilde{\pi}_{!} h_{!}\left(\Lambda_{Y}^{n}\right) \cong \tilde{\pi}_{*} h_{!}\left(\Lambda_{Y}^{n}\right),
$$

so $H_{\text {ét }}^{i}\left(X_{\widehat{K}}, \mathscr{G}\right) \cong H_{\text {êt }}^{i}\left(\tilde{Y}_{\bar{K}}, h_{!} \Lambda_{Y}\right)^{\oplus n}$. Now, writing $i: V \rightarrow \tilde{Y}$ for the closed complement of $Y$, we get exact sequences

$$
H_{\text {ét }}^{i-1}\left(V_{\widehat{\bar{K}}}, \Lambda_{V}\right) \rightarrow H_{\text {ét }}^{i}\left(\tilde{Y}_{\widehat{\bar{K}}}, h_{!} \Lambda_{Y}\right) \rightarrow H_{\text {ét }}^{i}\left(\tilde{Y}_{\widehat{\bar{K}}}, \Lambda_{\tilde{Y}}\right)
$$

for all $i$. Examining this sequence for any fixed $i>d$, we see that the rightmost term vanishes by Theorem 1.4, while the leftmost term vanishes by the assumption that $\mathcal{T}_{d-1, d-1}$ holds. ${ }^{8}$ Therefore $H_{\text {ét }}^{i}\left(\tilde{Y}_{\widehat{K}}, h_{!} \Lambda_{Y}\right)=0$ for $i>d$, as desired.

\footnotetext{
${ }^{6}$ This step was inspired by some constructions in Nori's beautiful paper [Nor02].

${ }^{7}$ Surjectivity here can be checked either by a direct calculation or by "pure thought" ( $\pi$ ! is left adjoint to $\pi *$, and left adjoints preserve epimorphisms).

${ }^{8}$ One really needs the induction hypothesis to control the leftmost term here, since $V$ may not be normal.
} 
Step Four. Suppose that $\mathcal{T}_{d-1, d-1}$ holds. Then $\mathcal{T}_{d, i+1}$ implies $\mathcal{T}_{d, i}$ for any $i \geq d$.

Fix $d$ and $i \geq d$ as in the statement, and assume $\mathcal{T}_{d, i+1}$ is true. Let $X$ be a $d$-dimensional affinoid, and let $\mathscr{F}$ be a Zariski-constructible sheaf on $X$. We need to show that $H_{\text {ét }}^{i+1}\left(X_{\widehat{K}}, \mathscr{F}\right)=0$. By Step Two, we can assume $X$ is normal (or even that $X$ is the $d$-dimensional affinoid ball). By Step One, it suffices to show that $H_{\text {ét }}^{i+1}\left(X_{\widehat{K}}, j ! j^{*} \mathscr{F}\right)=0$ where $j: U \rightarrow X$ is the inclusion of any dense Zariski-open subset. Fix a choice of such a $U$ with the property that $j^{*} \mathscr{F}$ is locally constant. By Step Three, we can choose a Zariski-constructible sheaf $\mathscr{G}$ on $X$ and a surjection $s: \mathscr{G} \rightarrow j ! j^{*} \mathscr{F}$ such that $H_{\mathrm{et}}^{n}\left(X_{\widehat{K}}, \mathscr{G}\right)=0$ for all $n>d$. By Proposition 2.4, the sheaf $\mathscr{K}=\operatorname{ker} s$ is Zariski-constructible. Now, looking at the exact sequence

$$
H_{\text {ét }}^{i+1}\left(X_{\widehat{K}}, \mathscr{G}\right) \rightarrow H_{\text {ét }}^{i+1}\left(X_{\widehat{K}}, j ! j^{*} \mathscr{F}\right) \rightarrow H_{\text {ét }}^{i+2}\left(X_{\widehat{K}}, \mathscr{K}\right),
$$

we see that the leftmost term vanishes by the construction of $\mathscr{G}$, while the rightmost term vanishes by the induction hypothesis. Therefore

$$
H_{\text {ét }}^{i+1}\left(X_{\widehat{K}}, j ! j^{*} \mathscr{F}\right)=0,
$$

as desired.

\subsection{Constant coefficients}

In this section we prove Theorem 1.4. The following technical lemma plays an important role in the argument.

Lemma 3.1. Let $K$ be a complete discretely valued nonarchimedean field, and let $A$ be a reduced $K$-affinoid algebra. Then $A^{\circ}$ is an excellent Noetherian ring. Moreover, the strict Henselization of any localization of $A^{\circ}$ is excellent as well.

Proof. By Noether normalization for affinoids and [BGR84, Corollary 6.4.1/6], $A^{\circ}$ can be realized as a module-finite integral extension of $\mathcal{O}_{K}\left\langle T_{1}, \ldots, T_{n}\right\rangle$ with $n=\operatorname{dim} A$. By a result of Valabrega (cf. [Val75, Proposition 7] and [Val76, Theorem 9]), the convergent power series ring $\mathcal{O}_{K}\left\langle T_{1}, \ldots, T_{n}\right\rangle$ is excellent for any complete discrete valuation ring $\mathcal{O}_{K}$. Since excellence propagates along finite type ring maps and localizations, cf. [Sta17, Tag 07QU], we see that $A^{\circ}$ and any localization thereof is excellent. Now, by a result of Greco [Gre76, Corollary 5.6.iii], the strict Henselization of any excellent local ring is excellent, which gives what we want.

We also need the following extremely powerful theorem of Gabber.

Theorem 3.2 (Gabber). Let $B$ be a quasi-excellent strictly Henselian local ring, and let $U \subset \operatorname{Spec} B$ be an affine open subscheme. Then $H_{\text {ét }}^{i}(U, \mathbf{Z} / n \mathbf{Z})=0$ for any $i>\operatorname{dim} B$ and any integer $n$ invertible in $B$.

Proof. This is a special case of Gabber's affine Lefschetz theorem for quasi-excellent schemes, cf. Corollaire XV.1.2.4 in [ILO14].

Finally, we recall the following strong form of the Artin-Grothendieck vanishing theorem [SGA73, §XIV.3].

Theorem 3.3 (Artin-Grothendieck). Let $X$ be an affine variety over a separably closed field $k$, and let $\mathscr{F}$ be a torsion abelian sheaf on $X_{\text {ét }}$. Set

$$
\delta(\mathscr{F})=\sup \left\{\operatorname{tr} \cdot \operatorname{deg} k(x) / k \mid \mathscr{F}_{\bar{x}} \neq 0\right\} .
$$

Then $H_{\text {êt }}^{i}(X, \mathscr{F})=0$ for all $i>\delta(\mathscr{F})$. 
Proof of Theorem 1.4. Let $X=$ Spa $A$ be a $K$-affinoid as in the theorem. After replacing $K$ by $\widehat{K^{\text {nr }}}$ and $X$ by $X_{\widetilde{K^{\text {nr }}}}^{\text {red }}$, we can assume that $A$ is reduced and that $K$ has separably closed residue field $k$. By an easy induction we can also assume that $n=l$ is prime. For notational simplicity we give the remainder of the proof in the case where $\operatorname{char}(k)=p>0$; the equal characteristic zero case is only easier.

By e.g. Corollary 2.4.6 in [Ber93], Gal ${ }_{\bar{K} / K}$ sits in a short exact sequence

$$
1 \rightarrow P \rightarrow \mathrm{Gal}_{\bar{K} / K} \rightarrow T \simeq \prod_{q \neq p} \mathbf{Z}_{q} \rightarrow 1
$$

where $P$ is pro- $p$. In particular, if $L \subset \bar{K}$ is any finite extension of $K$, then

$$
H^{i}\left(\mathrm{Gal}_{\bar{K} / L}, \mathbf{Z} / l \mathbf{Z}\right) \simeq\left\{\begin{array}{l}
\mathbf{Z} / l \mathbf{Z} \text { if } i=0,1 \\
0 \text { if } i>1
\end{array} .\right.
$$

For any such $L$, look at the Cartan-Leray spectral sequence

$$
E_{2}^{i, j}=H^{i}\left(\mathrm{Gal}_{\bar{K} / L}, H_{\text {ét }}^{j}\left(X_{\widehat{K}}, \mathbf{Z} / l \mathbf{Z}\right)\right) \Rightarrow H_{\text {ét }}^{i+j}\left(X_{L}, \mathbf{Z} / l \mathbf{Z}\right)
$$

The group $\oplus_{j \geq 0} H_{\text {ét }}^{j}\left(X_{\widehat{K}}, \mathbf{Z} / l \mathbf{Z}\right)$ is finite by vanishing in degrees $>2 \operatorname{dim} X$ together with [Ber15, Theorem 1.1.1], so all the Galois actions in the $E_{2}$-page are trivial for any large enough $L,{ }^{9}$ in which case we can rewrite the spectral sequence as

$$
E_{2}^{i, j}=H^{i}\left(\mathrm{Gal}_{\bar{K} / L}, \mathbf{Z} / l \mathbf{Z}\right) \otimes H_{\text {ét }}^{j}\left(X_{\widehat{K}}, \mathbf{Z} / l \mathbf{Z}\right) \Rightarrow H_{\text {ét }}^{i+j}\left(X_{L}, \mathbf{Z} / l \mathbf{Z}\right) .
$$

Now if $d$ is the largest integer such that $H_{\text {ét }}^{d}\left(X_{\widehat{K}}, \mathbf{Z} / l \mathbf{Z}\right) \neq 0$, then the $E_{2}^{1, d}$ term survives the spectral sequence, so $H_{\text {ét }}^{d+1}\left(X_{L}, \mathbf{Z} / l \mathbf{Z}\right) \neq 0$. Since the residue field of $L$ is still separably closed, it thus suffices to prove the following statement:

$(\dagger)$ For any reduced affinoid $X=\operatorname{Spa} A$ over a complete discretely valued nonarchimedean field $K$ with separably closed residue field $k$ of characteristic $p>0$, we have $H_{\text {ét }}^{i}(X, \mathbf{Z} / l \mathbf{Z})=0$ for all $i>1+\operatorname{dim} X$ and all primes $l \neq p$.

Fix a uniformizer $\varpi \in \mathcal{O}_{K}$. Set $\mathcal{X}=\operatorname{Spec} A^{\circ}$ and $\mathcal{X}_{s}=\operatorname{Spec} A^{\circ} / \varpi$, so $\mathcal{X}_{s}$ is an affine variety

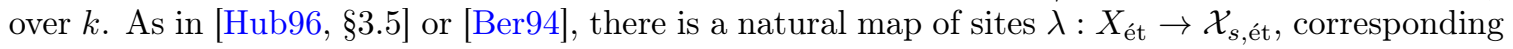
to the natural functor

$$
\begin{aligned}
\mathcal{X}_{s, \text { ét }} & \rightarrow X_{\text {ét }} \\
\mathcal{U} / \mathcal{X}_{s} & \mapsto \eta(\mathcal{U}) / X
\end{aligned}
$$

given by (uniquely) deforming an étale map $\mathcal{U} \rightarrow \mathcal{X}_{s}$ to a $\varpi$-adic formal scheme étale over $\operatorname{Spf} A^{\circ}$ and then passing to rigid generic fibers. (We follow Huber's notation in writing $\lambda$ - Berkovich denotes this map by $\Theta$.) For any abelian étale sheaf $\mathscr{F}$ on $X$, derived pushforward along $\lambda$ gives rise to the so-called nearby cycle sheaves $R^{j} \lambda_{*} \mathscr{F}$ on $\mathcal{X}_{s \text {,ét }}$, which can be calculated as the sheafifications of the presheaves $\mathcal{U} \mapsto H_{\text {ét }}^{j}(\eta(\mathcal{U}), \mathscr{F})$, and there is a spectral sequence

$$
H_{\text {ét }}^{i}\left(\mathcal{X}_{s}, R^{j} \lambda_{*} \mathscr{F}\right) \Rightarrow H_{\text {ét }}^{i+j}(X, \mathscr{F})
$$

\footnotetext{
${ }^{9}$ This trick was inspired by a discussion of Poincaré dualities in an IHES lecture by Peter Scholze, cf. https://www.youtube.com/watch?v=E3zAEqkd9cQ.
} 
cf. Proposition 4.1 and Corollary 4.2.(iii) in [Ber94]. Taking $\mathscr{F}=\mathbf{Z} / l \mathbf{Z}$, we see that to prove $(\dagger)$ it's enough to show that $H_{\text {ét }}^{i}\left(\mathcal{X}_{s}, R^{j} \lambda_{*} \mathbf{Z} / l \mathbf{Z}\right)=0$ for any $j \geq 0$ and any $i>1+\operatorname{dim} X-j$. By the strong form of the Artin-Grothendieck vanishing theorem recalled above, we're reduced to proving that if $x \in \mathcal{X}_{s}$ is any point such that

$$
\left(R^{j} \lambda_{*} \mathbf{Z} / l \mathbf{Z}\right)_{\bar{x}} \neq 0
$$

then tr.deg $k(x) / k \leq 1+\operatorname{dim} X-j$.

We check this by a direct computation. So, let $x \in \mathcal{X}_{s}$ be any point, and let $\mathfrak{p}_{x} \subset A^{\circ}$ be the associated prime ideal. Crucially, we have a "purely algebraic" description of the stalk $\left(R^{j} \lambda_{*} \mathbf{Z} / l \mathbf{Z}\right)_{\bar{x}}$ : letting $\mathcal{O}_{\mathcal{X}, \bar{x}}$ denote the strict Henselization of $\mathcal{O}_{\mathcal{X}, x}=\left(A^{\circ}\right)_{\mathfrak{p}_{x}}$ as usual, then

$$
\left(R^{j} \lambda_{*} \mathbf{Z} / l \mathbf{Z}\right)_{\bar{x}} \cong H_{\text {ét }}^{j}\left(\operatorname{Spec} \mathcal{O}_{\mathcal{X}, \bar{x}}\left[\frac{1}{\varpi}\right], \mathbf{Z} / l \mathbf{Z}\right) .
$$

This is a special case of [Hub96, Theorem 3.5.10], and it's remarkable that we have a description like this which doesn't involve taking some completion. By Lemma 3.1, $\mathcal{O}_{\mathcal{X}, \bar{x}}$ is excellent, so Theorem 3.2 implies that $H_{\text {ét }}^{j}(U, \mathbf{Z} / l \mathbf{Z})=0$ for any open affine subscheme $U \subset \operatorname{Spec} \mathcal{O}_{\mathcal{X}, \bar{x}}$ and any $j>\operatorname{dim} \mathcal{O}_{\mathcal{X}, \bar{x}}$. In particular, taking $U=\operatorname{Spec} \mathcal{O}_{\mathcal{X}, \bar{x}}\left[\frac{1}{\varpi}\right]$ and applying Huber's formula $(*)$ above, we see that if $j$ is an integer such that $\left(R^{j} \lambda_{*} \mathbf{Z} / l \mathbf{Z}\right)_{\bar{x}} \neq 0$, then necessarily

$$
j \leq \operatorname{dim} \mathcal{O}_{\mathcal{X}, \bar{x}}=\operatorname{dim} \mathcal{O}_{\mathcal{X}, x}=\operatorname{ht} \mathfrak{p}_{x},
$$

where the first equality follows from e.g. [Sta17, Tag 06LK]. Writing $R=A^{\circ} / \varpi$ and $\overline{\mathfrak{p}_{x}}=\mathfrak{p}_{x} / \varpi \subset R$, we then have

$$
\begin{aligned}
j+\operatorname{tr} \cdot \operatorname{deg} k(x) / k & \leq \operatorname{ht} \mathfrak{p}_{x}+\operatorname{tr} \cdot \operatorname{deg} k(x) / k \\
& =1+\operatorname{ht} \overline{\mathfrak{p}_{x}}+\operatorname{tr} \cdot \operatorname{deg} k(x) / k \\
& =1+\operatorname{dim} R_{\overline{\mathfrak{p}_{x}}}+\operatorname{dim} R / \overline{\mathfrak{p}_{x}} \\
& \leq 1+\operatorname{dim} R \\
& \leq 1+\operatorname{dim} X .
\end{aligned}
$$

Here the second line follows from the fact that $\varpi \in \mathfrak{p}_{x}$ is part of a system of parameters of $\left(A^{\circ}\right)_{\mathfrak{p}_{x}}$, so ht $\overline{\mathfrak{p}_{x}}=\operatorname{dim} R_{\overline{\mathfrak{p}_{x}}}=\operatorname{dim}\left(A^{\circ}\right)_{\mathfrak{p}_{x}}-1$; the third line is immediate from the equality $\operatorname{tr} . \operatorname{deg} k(x) / k=$ $\operatorname{dim} R / \overline{\mathfrak{p}_{x}}$, which is a standard fact about domains of finite type over a field; the fourth line is trivial; and the fifth line follows from the fact that $R$ is module-finite over $k\left[T_{1}, \ldots, T_{n}\right]$ with $n=\operatorname{dim} X$. But then

$$
\operatorname{tr} . \operatorname{deg} k(x) / k \leq 1+\operatorname{dim} X-j
$$

as desired.

\subsection{Stein spaces}

We end this section with the following slightly more general version of the vanishing theorem.

Corollary 3.4. Let $X$ be a rigid space over a characteristic zero complete discretely valued nonarchimedean field $K$ which is weakly Stein in the sense that it admts an admissible covering $X=$ $\cup_{n \geq 1} U_{n}$ by a nested sequence of open affinoid subsets $U_{1} \subset U_{2} \subset U_{3} \subset \cdots$. Let F्F be any Zariskiconstructible sheaf of $\mathbf{Z} / n \mathbf{Z}$-modules on $X_{\text {ét }}$ for some $n$ prime to the residue characteristic of $K$. Then

for all $i>\operatorname{dim} X$.

$$
H_{\text {ét }}^{i}\left(X_{\widehat{K}}, \mathscr{F}\right)=0
$$


Proof. By [Hub96, Lemma 3.9.2], we have a short exact sequence

$$
0 \rightarrow \lim _{\leftarrow n}^{1} H_{\text {ét }}^{i-1}\left(U_{n, \widehat{K}}, \mathscr{F}\right) \rightarrow H_{\text {êt }}^{i}\left(X_{\widehat{\bar{K}}}, \mathscr{F}\right) \rightarrow \lim _{\leftarrow n} H_{\text {êt }}^{i}\left(U_{n, \widehat{K}}, \mathscr{F}\right) \rightarrow 0 .
$$

But the groups $H_{\text {ét }}^{j}\left(U_{i, \widehat{K}}, \mathscr{F}\right)$ are finite, so the $\lim ^{1}$ term vanishes, and the result now follows from Theorem 1.3.

This argument also shows that Conjecture 1.2, for a fixed choice of $C$, is equivalent to the apparently more general conjecture that the cohomology of any Zariski-constructible sheaf on any weakly Stein space $X$ over $C$ vanishes in all degrees $>\operatorname{dim} X$.

\section{Comparison theorems}

\subsection{The affinoid comparison theorem}

In this section we prove Theorem 1.9. Note that when $\mathscr{F}$ is a constant sheaf of torsion abelian groups, this theorem is already in Huber's book [Hub96], and we'll eventually reduce to this case.

Let $\mathcal{S}$ and $S$ be as in the statement of the theorem. Before continuing, we note for later use that if $j: \mathcal{U} \rightarrow \mathcal{S}$ is any open immersion with closed complement $i: \mathcal{Z} \rightarrow \mathcal{S}$, the four functors $j_{!}, j^{*}$, $i_{*}, i^{*}$ and their analytifications can be canonically and functorially commuted with the appropriate $\mu^{*}$ 's in the evident sense. Indeed, for $j^{*}$ and $i^{*}$ this is obvious (by taking adjoints of the obvious equivalences $j_{*} \mu_{U *} \cong \mu_{S *} j_{*}^{\text {an }}$ and $\left.i_{*} \mu_{Z *} \cong \mu_{X *} i_{*}^{\text {an }}\right)$, for $j_{\text {! }}$ it follows by taking adjoints of the equivalence $j^{*} \mu_{S *} \cong \mu_{U *} j^{\text {an* }}$ (which holds by "base change for a slice"), and for $i_{*}$ it's a very special case of [Hub96, Theorem 3.7.2]. Moreover, if $f: \mathcal{Y}=\operatorname{Spec} \mathcal{B} \rightarrow \mathcal{S}$ is any finite morphism with analytification $f^{\text {an }}: Y=\operatorname{Spa} B \rightarrow X$, then $\mu_{X}^{*} f_{*} \cong f_{*}^{\text {an }} \mu_{Y}^{*}$ (by [Hub96, Theorem 3.7.2] and [Hub96, Proposition 2.6.4] again) and $\mu_{Y}^{*} f^{*} \cong f^{\text {an* }} \mu_{X}^{*}$ (by taking adjoints to the obvious equivalence $\left.\mu_{X *} f_{*}^{\text {an }} \cong f_{*} \mu_{Y *}\right)$. We'll use all of these compatibilities without further comment.

Proof of Theorem 1.9. First, observe that all functors involved in the statement of the theorem commute with filtered colimits: for $H_{\text {ét }}^{n}(\mathcal{S},-)$ this is standard, for $H_{\text {ét }}^{n}(S,-)$ this follows from [Hub96, Lemma 2.3.13], and for $\mu_{S}^{*}$ it is trivial (because $\mu_{S}^{*}$ is a left adjoint). Writing $\mathscr{F}$ as the filtered colimit of its $m$-torsion subsheaves, we therefore reduce to the case where $\mathscr{F}$ is killed by some integer $m \geq 1$. Since $\mathcal{S}$ is qcqs, we can write any sheaf of $\mathbf{Z} / m \mathbf{Z}$-modules on $\mathcal{S}_{\text {ét }}$ as a filtered colimit of constructible sheaves of $\mathbf{Z} / m \mathbf{Z}$-modules, cf. [Sta17, Tag 03SA], which reduces us further to the case where $\mathscr{F}$ is a constructible sheaf of $\mathbf{Z} / m \mathbf{Z}$-modules.

Next, by repeated application of [SGA73, Prop. IX.2.14(ii)], we may choose a resolution

$$
\mathscr{F} \simeq\left[\mathscr{G}^{0} \rightarrow \mathscr{G}^{1} \rightarrow \mathscr{G}^{2} \rightarrow \cdots\right]
$$

where each sheaf $\mathscr{G}^{i}$ is isomorphic to a finite direct sum of sheaves of the form $\pi_{*} \underline{M}$, where $\pi: \mathcal{S}^{\prime} \rightarrow \mathcal{S}$ is a finite morphism and $M$ is a finite $\mathbf{Z} / n \mathbf{Z}$-module. By Lemma 4.1 , it then suffices to prove that $H_{\text {ét }}^{n}\left(\mathcal{S}, \pi_{*} \underline{M}\right) \rightarrow H_{\text {ét }}^{n}\left(S, \mu_{S}^{*} \pi_{*} \underline{M}\right)$ is an isomorphism for any such $\pi$ and $M$, and any $n$. Using the exactness of $\pi_{*}$ and $\pi_{*}^{\text {an }}$ and their compatibility with $\mu$-pullback, one immediately reduces further to the case of constant sheaves, which is exactly [Hub96, Corollary 3.2.3].

In the previous argument we used the following lemma, which is less trivial than it appears. The reader may wish to skip the proof. 
Lemma 4.1. Let $f: \mathcal{D} \rightarrow \mathcal{C}$ be a morphism of sites, and let $\mathscr{F} \bullet$ be a bounded-below complex of abelian sheaves on $\mathcal{C}$. Then there is a map of spectral sequences

$$
\begin{array}{ccc}
E_{1}^{i, j}=H^{j}\left(\mathcal{C}, \mathscr{F}^{i}\right) & \Rightarrow H^{i+j}\left(\mathcal{C}, \mathscr{F}^{\bullet}\right) \\
& \Downarrow \\
E_{1}^{i, j}=H^{j}\left(\mathcal{D}, f^{-1} \mathscr{F}^{i}\right) & \Rightarrow H^{i+j}\left(\mathcal{D}, f^{-1} \mathscr{F}^{\bullet}\right)
\end{array}
$$

which is given on the $E_{1}$-page and abutment by the canonical maps $H^{j}\left(\mathcal{C}, \mathscr{F}^{i}\right) \rightarrow H^{j}\left(\mathcal{D}, f^{-1} \mathscr{F}^{i}\right)$ and $H^{j}(\mathcal{C}, \mathscr{F} \bullet) \rightarrow H^{j}\left(\mathcal{D}, f^{-1} \mathscr{F} \bullet\right)$, respectively.

Proof. Filter $\mathscr{F}^{\bullet}$ by the subcomplexes $F^{i} \mathscr{F}^{\bullet}=\left[\mathscr{F}^{i} \rightarrow \mathscr{F}^{i+1} \rightarrow \cdots\right]$. By [Sta17, 0BKI], we can choose a filtered complex $\mathscr{G}^{\bullet}$ of abelian sheaves on $\mathcal{C}$ together with a filtered quasi-isomorphism $\alpha: \mathscr{F}^{\bullet} \rightarrow \mathscr{G}^{\bullet}$ such that (for all $j$ ) $\mathscr{G}^{\bullet}$ and $\operatorname{gr}^{j} \mathscr{G}^{\bullet}$ are K-injective complexes of injective sheaves and

$$
\operatorname{gr}^{j} \alpha: \operatorname{gr}^{j} \mathscr{F}^{\bullet}=\mathscr{F}^{j}[-j] \rightarrow \operatorname{gr}^{j} \mathscr{G}^{\bullet}
$$

is a quasiisomorphism. Unwinding definitions, one checks that the upper spectral sequence in question coincides with the spectral sequence associated with the filtered complex $\Gamma\left(\mathcal{C}, \mathscr{G}^{\bullet}\right)$.

Applying [Sta17, 0BKI] again, we can choose a filtered complex $\mathscr{H}^{\bullet}$ of abelian sheaves on $\mathcal{D}$ together with a filtered quasi-isomorphism $\beta: f^{-1} \mathscr{G}^{\bullet} \rightarrow \mathscr{H}^{\bullet}$ such that (for all $j$ ) $\mathscr{H}^{\bullet}$ and $\mathrm{gr}^{j} \mathscr{H}^{\bullet}$ are $\mathrm{K}$-injective complexes of injective sheaves and $\operatorname{gr}^{j} \beta$ is a quasi-isomorphism. Again, one checks directly that the lower spectral sequence identifies with the spectral sequence associated with the filtered complex $\Gamma\left(\mathcal{D}, \mathscr{H}^{\bullet}\right)$. By construction, we have filtered maps of filtered complexes

$$
\Gamma\left(\mathcal{C}, \mathscr{G}^{\bullet}\right) \rightarrow \Gamma\left(\mathcal{D}, f^{-1} \mathscr{G}^{\bullet}\right) \rightarrow \Gamma\left(\mathcal{D}, \mathscr{H}^{\bullet}\right)
$$

where the lefthand map exists by general nonsense and the righthand map is induced by $\beta$. The composition of these maps induces the desired map of spectral sequences.

\subsection{The relative comparison theorem}

In this section we prove Theorem 1.8. Fix $S, \mathcal{S}$ and $\Lambda$ as in the theorem. In what follows we will write $\mathcal{X}, \mathcal{Y}, \mathcal{U}$, etc. for schemes locally of finite type over $\mathcal{S}$, and $X, Y, U$, etc. for the corresponding rigid analytic spaces over $S$. If $f: \mathcal{X} \rightarrow \mathcal{Y}$ is a finite type morphism as in the theorem, then for any $F \in D_{c}^{+}(\mathcal{X}, \Lambda)$ we write $\operatorname{comp}(f, F)$ for the natural base change map $\mu_{Y}^{*} R f_{*} F \rightarrow R f_{*}^{\text {an }} \mu_{X}^{*} F$. For brevity, we will say that "comp $(f)$ is an isomorphism" if the map $\operatorname{comp}(f, F)$ is an isomorphism in $D(Y, \Lambda)$ for every $F \in D_{c}^{+}(\mathcal{X}, \Lambda)$. (In general, it is not true that the base change map $\mu_{Y}^{*} R f_{*} \rightarrow$ $R f_{*}^{a n} \mu_{X}^{*}$ is a natural isomorphism of functors.)

Before beginning the proof, we collect some preliminary observations. We will freely use the following result, which is well-known to experts.

Proposition 4.2 (Grothendieck, Gabber). Let $f: \mathcal{X} \rightarrow \mathcal{Y}$ be a finite type morphism between quasiexcellent Noetherian schemes, and let $\Lambda$ be a Noetherian ring killed by some positive integer invertible on $\mathcal{Y}$. Then the functor $R f_{*}: D(\mathcal{X}, \Lambda) \rightarrow D(\mathcal{Y}, \Lambda)$ preserves constructibility; more precisely, it carries $D_{c}^{b}(\mathcal{X}, \Lambda)$ into $D_{c}^{b}(\mathcal{Y}, \Lambda)$.

Proof. Cover $\mathcal{X}$ by open affines $\mathcal{X}_{i}, 0 \leq i \leq n$; for any $0 \leq i_{1}<\cdots<i_{p} \leq n$, set $\mathcal{X}_{i_{1}<\cdots<i_{p}}=$ $\cap_{1 \leq j \leq p} \mathcal{X}_{i_{j}}$, so each $\mathcal{X}_{i_{1}<\cdots<i_{p}}$ is a separated open subscheme of $\mathcal{X}$. Let $f_{i_{1}<\cdots<i_{p}}: \mathcal{X}_{i_{1}<\cdots<i_{p}} \rightarrow \mathcal{Y}$ be the evident morphism. Looking at the the Cech spectral sequence

$$
E_{1}^{p, q}=\left.\oplus_{i_{1}<\cdots<i_{p}} R^{q}\left(f_{i_{1}<\cdots<i_{p}}\right)_{*} F\right|_{\mathcal{X}_{i_{1}<\cdots<i_{p}}} \Rightarrow R^{p+q} f_{*} F
$$


it suffices to show that each $R\left(f_{i_{1}<\cdots<i_{p}}\right)_{*}$ sends $D_{c}^{b}\left(\mathcal{X}_{i_{1}<\cdots<i_{p}}, \Lambda\right)$ into $D_{c}^{b}(\mathcal{Y}, \Lambda)$. In particular, it suffices to consider the case where $\mathcal{X}$ is separated. Since $f$ is then separated, by Nagata's compactification theorem we may choose a factorization $f=\bar{f} \circ j$ where $\bar{f}: \mathcal{X}^{\prime} \rightarrow \mathcal{Y}$ is proper and $j: \mathcal{X} \rightarrow \mathcal{X}^{\prime}$ is an open immersion. Combining [ILO14, Theorem XIII.1.1.1] and [ILO14, Proposition XVII.7.6.7], we see that $R j_{*}$ sends $D_{c}^{b}(\mathcal{X}, \Lambda)$ into $D_{c}^{b}\left(\mathcal{X}^{\prime}, \Lambda\right)$. By [SGA73, Th. XIV.1.1], $R \bar{f}_{*}$ sends $D_{c}^{b}\left(\mathcal{X}^{\prime}, \Lambda\right)$ into $D_{c}^{b}(\mathcal{Y}, \Lambda)$. Since $R f_{*} \cong R \bar{f}_{*} R j_{*}$, this gives the result.

Proposition 4.3. Let $h: \mathcal{X} \rightarrow \mathcal{Y}$ and $g: \mathcal{Y} \rightarrow \mathcal{Z}$ be finite type morphisms of locally finite type $\mathcal{S}$-schemes.

i. If $\operatorname{comp}(h)$ and $\operatorname{comp}(g)$ are isomorphisms, then so is $\operatorname{comp}(g \circ h)$.

ii. If $\operatorname{comp}(h)$ and $\operatorname{comp}(g \circ h)$ are isomorphisms, then is $\operatorname{comp}\left(g, R h_{*} F\right)$ is an isomorphism for every $F \in D_{c}^{+}(\mathcal{X}, \Lambda)$.

iii. (2-out-of-3 property) If $F_{1} \rightarrow F_{2} \rightarrow F_{3} \rightarrow$ is a distinguished triangle in $D(\mathcal{X}, \Lambda)$ such that two of the three maps $\operatorname{comp}\left(h, F_{i}\right)$ are isomorphisms, then so is the third.

Proof. Part i. is a special case of the composability of base change maps, cf. [Sta17, Tag 0E46]. For ii., consider the composition

$$
\mu_{Z}^{*} R(g \circ h)_{*} F \cong \mu_{Z}^{*} R g_{*} R h_{*} F \rightarrow R g_{*}^{\text {an }} \mu_{Y}^{*} R h_{*} F \rightarrow R g_{*}^{\text {an }} R h_{*}^{\text {an }} \mu_{X}^{*} F \cong R(g \circ h)_{*}^{\text {an }} \mu_{X}^{*} F,
$$

where the first arrow is $\operatorname{comp}\left(g, R h_{*} F\right)$ and the second arrow is $R g_{*}^{\text {an }} \operatorname{comp}(h, F)$. By the composability of base change maps, this composition is the comparison map $\operatorname{comp}(g \circ h, F)$. By our assumptions, the second arrow and the composition of both arrows are isomorphisms. We then conclude by observing that if $A \stackrel{s}{\rightarrow} B \stackrel{t}{\rightarrow} C$ is any pair of morphisms in any category such that $t$ and $t \circ s$ are isomorphisms, then $s$ is an isomorphism as well.

Part iii. is an easy exercise, using the fact that the three maps $\operatorname{comp}\left(h, F_{i}\right)$ define a morphism between the evident distinguished triangles.

Proposition 4.4. Suppose that $\operatorname{comp}(j)$ is an isomorphism for all open immersions $j: \mathcal{X} \rightarrow \mathcal{Y}$ of locally finite type $\mathcal{S}$-schemes. Then Theorem 1.8 is true.

Proof. First we show that if $\operatorname{comp}(j)$ is an isomorphism for all open immersions, then $\operatorname{comp}(f)$ is an isomorphism for all separated maps. For this, let $f: \mathcal{X} \rightarrow \mathcal{Y}$ be as in Theorem 1.8, and suppose that $f$ is separated. We can argue locally on $\mathcal{Y}$, so we can assume that $\mathcal{Y}$ is affine. By Nagata's compactification theorem we may choose a factorization $f=\bar{f} \circ j$ where $\bar{f}: \mathcal{X}^{\prime} \rightarrow \mathcal{Y}$ is proper and $j: \mathcal{X} \rightarrow \mathcal{X}^{\prime}$ is an open immersion. Then $\operatorname{comp}(j)$ is an isomorphism by assumption, and $\operatorname{comp}(\bar{f})$ is an isomorphism by Berkovich and Huber's proof of the proper case. We then conclude by part i. of the previous proposition.

For the general case, we can assume that $\mathcal{Y}$ is affine, so $\mathcal{X}$ is quasicompact. We now argue by induction on the minimal number $s(\mathcal{X})$ of separated open subschemes required to cover $\mathcal{X}$. Choose an open cover $\mathcal{X}=\mathcal{U} \cup \mathcal{V}$ where $\mathcal{U}$ is separated and $s(\mathcal{V})=s(\mathcal{X})-1$. Note that $\mathcal{U} \cap \mathcal{V}$ is separated. Let $j_{\mathcal{U}}: \mathcal{U} \rightarrow \mathcal{X}, j_{\mathcal{V}}: \mathcal{V} \rightarrow \mathcal{X}$, and $j_{\mathcal{U} \cap \mathcal{V}}: \mathcal{U} \cap \mathcal{V} \rightarrow \mathcal{X}$ be the evident open inclusions, so we get a functorial distinguished triangle

$$
F \rightarrow R j_{\mathcal{U} *} j_{\mathcal{U}}^{*} F \oplus R j_{\mathcal{V} *} j_{\mathcal{V}}^{*} F \rightarrow R j_{\mathcal{U} \cap \mathcal{V} *} j_{\mathcal{U} \cap \mathcal{V}}^{*} F \rightarrow
$$


for any $F \in D_{c}^{+}(\mathcal{X}, \Lambda)$. Applying $R f_{*}$, the comparison maps $\operatorname{comp}(f,-)$ fit together into a morphism

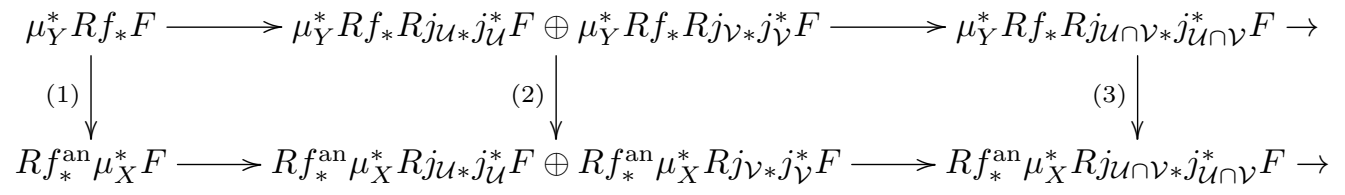

of distinguished triangles. For each $\bullet \in\{\mathcal{U}, \mathcal{V}, \mathcal{U} \cap \mathcal{V}\}$, the maps $\operatorname{comp}\left(j_{\bullet}\right)$ are isomorphisms by assumption; by induction on $s$, the maps $\operatorname{comp}\left(f \circ j_{\bullet}\right)$ are isomorphisms as well. Applying part ii. of the previous proposition with $g=f$ and $h=j_{\bullet}$, we see that the maps (2) and (3) are both isomorphisms. By the 2-out-of-3 property, the morphism (1) is thererfore an isomorphism, as desired.

Proposition 4.5. Suppose that $\operatorname{comp}(h, \underline{M})$ is an isomorphism for any open immersion $h: \mathcal{U} \rightarrow \mathcal{V}$ of locally finite type $\mathcal{S}$-schemes and any constant constructible sheaf of $\Lambda$-modules $\underline{M}$ on $\mathcal{U}$. Then Theorem 1.8 is true.

Proof. By the previous proposition, it suffices to deduce under these assumptions that $\operatorname{comp}(j, F)$ is an isomorphism for any open immersion $j: \mathcal{X} \rightarrow \mathcal{Y}$ and any $F \in D_{c}^{+}(\mathcal{X}, \Lambda)$. We can clearly assume that $\mathcal{Y}$ is affine and that $j$ has dense image. We can also assume that $F=\mathscr{F}$ is a constructible sheaf (placed in degree zero). As in the proof of Theorem 1.9, we may choose an isomorphism

$$
\mathscr{F} \simeq\left[\mathscr{G}^{0} \rightarrow \mathscr{G}^{1} \rightarrow \mathscr{G}^{2} \rightarrow \cdots\right]
$$

where each sheaf $\mathscr{G}^{i}$ is isomorphic to a finite direct sum of sheaves of the form $\pi_{*} \underline{M}$, where $\pi: \mathcal{U} \rightarrow \mathcal{X}$ is a finite morphism and $\underline{M}$ is a constant constructible sheaf of $\Lambda$-modules on $\mathcal{U}$. By an easy variant of the spectral sequence argument used in the proof of Theorem 1.9, it suffices to prove that $\operatorname{comp}\left(j, \mathscr{G}^{i}\right)$ is an isomorphism for all $i$. In particular, it suffices to prove that $\operatorname{comp}\left(j, \pi_{*} \underline{M}\right)$ is an isomorphism for any finite morphism $\pi: \mathcal{U} \rightarrow \mathcal{X}$ and any constant constructible sheaf $\underline{M}$ as above.

Fix such a $\pi$ and $\underline{M}$. By Zariski's main theorem, we can choose a commutative diagram

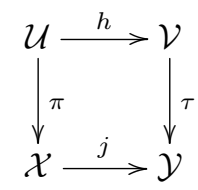

where $h$ is an open immersion and $\tau$ is finite. In what follows we freely use the fact that $\pi_{*}=R \pi_{*}$ and $\tau=R \tau_{*}$, and likewise for $\pi^{\text {an }}$ and $\tau^{\text {an }}$; we also freely use the fact that $\operatorname{comp}(\pi)$ is an isomorphism for any finite morphism $\pi$. Consider the sequence of canonical isomorphisms

$$
\begin{aligned}
\mu_{Y}^{*} R j_{*} \pi_{*} \underline{M} & \cong \mu_{Y}^{*} R(j \circ \pi)_{*} \underline{M} \\
& \cong \mu_{Y}^{*} R(\tau \circ h)_{*} \underline{M} \\
& \cong \mu_{Y}^{*} \tau_{*} R h_{*} \underline{M} \\
& \cong \tau_{*}^{\text {an }} \mu_{V}^{*} R h_{*} \underline{M} \\
& \stackrel{*}{\cong} \tau_{*}^{\text {an }} R h_{*}^{\text {an }} \mu_{U}^{*} \underline{M} \\
& \cong R j_{*}^{\text {an }} \pi_{*}^{\text {an }} \mu_{U}^{*} \underline{M} \\
& \cong R j_{*}^{\text {an }} \mu_{X}^{*} \pi_{*} \underline{M},
\end{aligned}
$$


where the starred isomorphism is the map $\tau_{*}^{\text {an }} \operatorname{comp}(h, \underline{M})$. One checks directly that the composition of these maps coincides with $\operatorname{comp}\left(j, \pi_{*} \underline{M}\right)$, so the latter is an isomorphism, as desired.

Proposition 4.6. Suppose that $\operatorname{comp}(h, \underline{M})$ is an isomorphism for any open immersion $h: \mathcal{X} \rightarrow \mathcal{Y}$ of regular finite type $\mathcal{S}$-schemes and any constant constructible sheaf $\underline{M}$ of $\Lambda$-modules. Then Theorem 1.8 is true.

Proof. By the previous proposition, it suffices to show that the hypothesis of the proposition implies that $\operatorname{comp}(j, F)$ is an isomorphism for any open immersion $j: \mathcal{X} \rightarrow \mathcal{Y}$ of finite type $\mathcal{S}$-schemes and any constant constructible sheaf $\underline{M}$ on $\mathcal{X}$.

Fix such a choice of $j$ and $\underline{M}$. We can clearly assume that $\mathcal{Y}$ is affine. By the topological invariant of the étale site, we can also assume that $\mathcal{Y}$ and $\mathcal{X}$ are reduced. Choose a dense open regular subscheme $g: \mathcal{U} \rightarrow \mathcal{X}$ with closed complement $i: \mathcal{Z} \rightarrow \mathcal{X}$, so we get a distinguished triangle

$$
i_{*} i^{!} \underline{M} \rightarrow \underline{M} \rightarrow R g_{*} g^{*} \underline{M} \rightarrow .
$$

By induction on dim supp, we can assume that $\operatorname{comp}\left(j, i_{*} i^{!} \underline{M}\right)$ is an isomorphism. By the 2-out-of-3 property, it now suffices to prove that $\operatorname{comp}\left(j, R g_{*} g^{*} \underline{M}\right)$ is an isomorphism. By another application of Proposition 4.3.ii, we observe that the latter map is an isomorphism if both $\operatorname{comp}\left(g, g^{*} \underline{M}\right)$ and $\operatorname{comp}\left(j \circ g, g^{*} \underline{M}\right)$ and are isomorphisms. In particular, we're reduced to showing that $\operatorname{comp}(h, \underline{M})$ is an isomorphism for any open immersion $h: \mathcal{X} \rightarrow \mathcal{Y}$ of reduced finite type $\mathcal{S}$-schemes with $\mathcal{X}$ regular.

Since $\mathcal{Y}$ is regular, applying the results of [Tem17] we can choose a commutative diagram

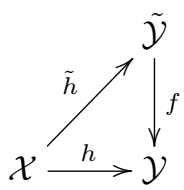

where $\tilde{h}$ is a dense open embedding of regular finite type $\mathcal{S}$-schemes and $f$ is a proper birational morphism. In particular, $\operatorname{comp}(f)$ is an isomorphism. By the hypotheses of the proposition, $\operatorname{comp}(\tilde{h}, \underline{M})$ is an isomorphism as well. Arguing as in the proof of Proposition 4.3.ii, one deduces that $\operatorname{comp}(h, \underline{M})$ is an isomorphism.

Proposition 4.7. Let $S, \mathcal{S}$ and $\Lambda$ be as in the statement of Theorem 1.8. Then for any open immersion $h: \mathcal{X} \rightarrow \mathcal{Y}$ of regular finite type $\mathcal{S}$-schemes and any constant constructible sheaf of $\Lambda$-modules $\underline{M}$, the map $\operatorname{comp}(h, \underline{M})$ is an isomorphism.

Proof. This follows exactly as in the final stages of the proof of [SGA73, Th. XVI.4.1], with the appeal to results in [SGA73, §XVI.3] replaced by Huber's cohomological purity theorem [Hub96, Theorem 3.9.1]

Putting together the previous two propositions, Theorem 1.8 follows. 


\subsection{Applications}

Proof of Theorem 1.10. For the first part of i., we compute that

$$
\begin{aligned}
R \Gamma(\mathcal{X}, F) & \stackrel{(1)}{\cong} R \Gamma\left(\mathcal{S}, R f_{*} F\right) \\
& \stackrel{(2)}{\cong} R \Gamma\left(S, \mu_{S}^{*} R f_{*} F\right) \\
& \stackrel{(3)}{\cong} R \Gamma\left(S, R f_{*}^{\text {an }} \mu_{X}^{*} F\right) \\
& \stackrel{(4)}{\cong} R \Gamma\left(X, \mu_{X}^{*} F\right)
\end{aligned}
$$

where (1) and (4) are the evident Leray isomorphisms, (2) follows from Theorem 1.9, and (3) follows from Theorem 1.8. For the second part of i., it suffices to assume that $F=\mathscr{F}[0]$ is a sheaf. In this case, we need to prove that $\mathscr{F} \stackrel{\sim}{\rightarrow} \mu_{X *} \mu_{X}^{*} \mathscr{F}$ and that $R^{i} \mu_{X *} \mu_{X}^{*} \mathscr{F}=0$ for all $i \geq 1$. By definition, $R^{i} \mu_{X *} \mu_{X}^{*} \mathscr{F}$ is the sheafification of the presheaf sending any finite type $\mathcal{S}$-scheme $\mathcal{U}$ with an étale map $g: \mathcal{U} \rightarrow \mathcal{X}$ to $H_{\text {ét }}^{i}\left(U, g^{\text {an* }} \mu_{X}^{*} \mathscr{F}\right)$, where of course $U=\mathcal{U}^{\text {an }}$ is the analytification of $\mathcal{U}$. But then

$$
H_{\text {ét }}^{i}\left(U, g^{\mathrm{an} *} \mu_{X}^{*} \mathscr{F}\right) \cong H_{\text {ét }}^{i}\left(U, \mu_{U}^{*} g^{*} \mathscr{F}\right) \cong H_{\text {ét }}^{i}\left(\mathcal{U}, g^{*} \mathscr{F}\right),
$$

where the second isomorphism follows from the first part of i., so the result follows from the locality of cohomology (cf. [Sta17, Tag 01FW]).

For ii., note that

$$
\begin{aligned}
\operatorname{Hom}_{D(X, \Lambda)}\left(\mu_{X}^{*} G, \mu_{X}^{*} F\right) & \cong \operatorname{Hom}_{D(\mathcal{X}, \Lambda)}\left(G, R \mu_{X *} \mu_{X}^{*} F\right) \\
& \cong \operatorname{Hom}_{D(\mathcal{X}, \Lambda)}(G, F),
\end{aligned}
$$

where the first isomorphism follows from the adjointness of $\mu_{X}^{*}$ and $R \mu_{X *}$, and the second isomorphism follows from part i.

Next, we prove the structure theorem.

Proof of Theorem 1.7. Full faithfulness is immediate from Theorem 1.10.ii together with the natural identification $\operatorname{Hom}_{\mathcal{A}}(M, N)=\operatorname{Hom}_{D(\mathcal{A})}(M, N)$, which holds for any abelian category $\mathcal{A}$.

For essential surjectivity, we argue as follows. By the topological invariance of étale sites, we can assume that $A$ is reduced. Fix $\mathscr{F} \in \operatorname{Sh}_{z c}(S, \Lambda)$. Pick a dense Zariski-open subset $j: U \rightarrow S$ such that $U$ is normal and $j^{*} \mathscr{F}$ is locally constant, and let $i: Z \rightarrow S$ be the inclusion of the closed complement. Let $j^{\text {alg }}: \mathcal{U} \rightarrow \mathcal{S}$ and $i^{\text {alg }}: \mathcal{Z} \rightarrow \mathcal{S}$ be the associated open and closed subschemes of $\mathcal{S}$. By induction on $\operatorname{dim} \operatorname{supp} \mathscr{F}$, we can assume that $i_{*} i^{*} \mathscr{F} \simeq \mu_{S}^{*} \mathcal{G}$ for some constructible sheaf $\mathcal{G}$. By Corollary 2.16, analytification induces an equivalence of categories $\mathcal{U}_{\text {fét }} \cong U_{\text {fét }}$. Since (on individual connected components of $U) j^{*} \mathscr{F}$ corresponds to a representation of $\pi_{1}(U, \bar{x}) \cong \pi_{1}(\mathcal{U}, \bar{x})$, we immediately see that $j^{*} \mathscr{F} \simeq \mu_{U}^{*} \mathcal{F}^{\prime}$ for some locally constant constructible sheaf $\mathcal{F}^{\prime}$ on $\mathcal{U}$, so then

$$
j_{!} j^{*} \mathscr{F} \simeq j_{!} \mu_{U}^{*} \mathcal{F}^{\prime} \cong \mu_{S}^{*} j_{!}^{\operatorname{alg}} \mathcal{F}^{\prime}=\mu_{S}^{*} \mathcal{F}
$$

where we've set $\mathcal{F}=j_{!}^{\text {alg }} \mathcal{F}^{\prime}$. Thus $\mathscr{F}$ sits in a short exact sequence

$$
0 \rightarrow \mu_{S}^{*} \mathcal{F} \rightarrow \mathscr{F} \rightarrow \mu_{S}^{*} \mathcal{G} \rightarrow 0
$$


To conclude, recall that for any abelian category $\mathcal{A}$, there is a canonical identification $\operatorname{Ext}_{\mathcal{A}}^{i}(B, A)=$ $\operatorname{Hom}_{D(\mathcal{A})}(B, A[i])$ for any objects $A, B$ in $\mathcal{A}$, cf. [Sta17, Tag 06XP]. Combining this observation with Theorem 1.10.ii., we conclude that $\mu_{S}^{*}$ induces a bijection

$$
\operatorname{Ext}_{\operatorname{Sh}(\mathcal{S}, \Lambda)}^{1}(\mathcal{G}, \mathcal{F}) \rightarrow \operatorname{Ext}_{\operatorname{Sh}(S, \Lambda)}^{1}\left(\mu_{S}^{*} \mathcal{G}, \mu_{S}^{*} \mathcal{F}\right)
$$

on Yoneda Ext groups. Therefore $\mathscr{F} \simeq \mu_{S}^{*} \mathcal{H}$ for some constructible sheaf $\mathcal{H}$ on $\mathcal{S}$, as desired.

Finally, we prove Theorem 1.11.

Proof of Theorem 1.11. For part i., let $j^{\text {alg }}: \mathcal{U} \rightarrow \mathcal{S}$ be the evident open immersion with analytification $j$. By Theorem 1.7, we can write $\mathscr{F} \simeq \mu_{U}^{*} \mathcal{F}$ for some $\mathcal{F} \in \operatorname{Sh}_{c}(\mathcal{U}, \Lambda)$. (Precisely, Theorem 1.7 produces a sheaf $\mathcal{G} \in \operatorname{Sh}_{c}(\mathcal{S}, \Lambda)$ with $j_{!} \mathscr{F} \simeq \mu_{S}^{*} \mathcal{G}$; we then take $\mathcal{F}=j^{\text {alg* }} \mathcal{G}$.) By Proposition 4.2, $R j_{*}^{\text {alg }} \mathcal{F}$ is constructible, so

$$
R j_{*} \mathscr{F} \cong R j_{*} \mu_{U}^{*} \mathcal{F} \cong \mu_{S}^{*} R j_{*}^{\text {alg }} \mathcal{F}
$$

is Zariski-constructible by Theorem 1.8 .

For part ii., the spectral sequence

$$
E_{p, q}^{2}=\oplus_{i+j=q} \operatorname{Tor}_{p}^{\Lambda}\left(\mathscr{H}^{-i}(\mathscr{F}), \mathscr{H}^{-j}(\mathscr{G})\right) \Rightarrow \mathscr{H}^{-p-q}\left(\mathscr{F} \otimes_{\Lambda}^{\mathbf{L}} \mathscr{G}\right)
$$

shows that any given cohomology sheaf $\mathscr{H}^{n}\left(\mathscr{F} \otimes_{\Lambda}^{\mathbf{L}} \mathscr{G}\right)$ is unchanged if we replace $\mathscr{F}$ by $\tau^{\geq-n} \mathscr{F}$ for some large $n \gg 0$. This reduces us to the case where $\mathscr{F}$ is bounded, and then (by an easy induction using the two-out-of-three property) to the case where $\mathscr{F}$ is a sheaf. Repeating this argument, we can also assume that $\mathscr{G}$ is a sheaf. Going to a stratification $S=\amalg S_{i}$ such that $\mathscr{F}$ and $\mathscr{G}$ are locally constant on each stratum, we reduce to the case where both sheaves are locally constant, which is easy.

For part iii., note that $\mathscr{H}^{n}\left(\mathbf{D}_{S} \mathscr{F}\right)$ is the sheafification of the presheaf sending any quasicompact separated étale map $j: U \rightarrow S$ to the module

$$
H^{n}\left(R \mathscr{H} \operatorname{om}_{\Lambda}\left(R \Gamma_{c}\left(U, j^{*} \mathscr{F}\right), \Lambda\right)\right) .
$$

Our assumptions on $K$ and $\Lambda$ guarantee that $R \Gamma_{c}(U,-)$ sends $D^{[a, b]}(U, \Lambda)$ into $D^{[a, b+N]}(\Lambda)$ for some $N$ depending only on the pair $(S, \Lambda)$, and that $R \mathscr{H} \operatorname{om}_{\Lambda}(-, \Lambda)$ sends $D^{[a, b]}(\Lambda)$ into $D^{[-b,-a+\operatorname{dim} \Lambda]}(\Lambda)$. This shows that $\mathbf{D}_{S}$ exchanges the boundedness conditions as claimed, and that any given cohomology sheaf $\mathscr{H}^{n}\left(\mathbf{D}_{S} \mathscr{F}\right)$ depends only on the truncation $\tau^{\leq b} \tau^{\geq a} \mathscr{F}$ for some large $a \ll 0 \ll b$. For the Zariski-constructibility of $\mathbf{D}_{S} \mathscr{F}$, we can therefore assume that $\mathscr{F}$ is bounded, and then that $\mathscr{F}$ is a sheaf. Pick a smooth dense Zariski-open $j: U \rightarrow S$ with closed (affinoid) complement $i: Z \rightarrow X$, so $j^{*} \omega_{S}=\omega_{U}$ is locally constant up to a shift and twist. Shrinking $U$ further, if necessary, we can also assume that $j^{*} \mathscr{F}$ is locally constant, so

$$
j^{*} \mathbf{D}_{S} \mathscr{F} \cong \mathbf{D}_{U} j^{*} \mathscr{F}=R \mathscr{H} \operatorname{om}_{U}\left(j^{*} \mathscr{F}, \omega_{U}\right)
$$

is locally constant constructible, up to a shift and twist. Dualizing the distinguished triangle

$$
j ! j^{*} \mathscr{F} \rightarrow \mathscr{F} \rightarrow i_{*} i^{*} \mathscr{F} \rightarrow
$$

and using the habitual isomorphisms $\mathbf{D}_{S} j_{!} \cong R j_{*} \mathbf{D}_{U}$ and $\mathbf{D}_{S} i_{*} i^{*} \cong i_{*} \mathbf{D}_{Z} i^{*}$, we get a distinguished triangle

$$
i_{*} \mathbf{D}_{Z} i^{*} \mathscr{F} \rightarrow \mathbf{D}_{S} \mathscr{F} \rightarrow R j_{*} \mathbf{D}_{U} j^{*} \mathscr{F} \rightarrow .
$$


By induction on $\operatorname{dim} S$, the first term in this triangle is Zariski-constructible. Since $\mathbf{D}_{U} j^{*} \mathscr{F}$ is locally constant, the third term is Zariski-constructible by part i. The result now follows from the two-out-of-three property. Finally, for the reflexivity of $\mathscr{F}$, we can again assume that $\mathscr{F}$ is a sheaf, in which case the desired result is [Han17, Theorem B.1.2].

For part iv., note that we have isomorphisms

$$
\begin{aligned}
R \mathscr{H} \operatorname{om}_{S}(\mathscr{F}, \mathscr{G}) & \cong R \mathscr{H} \operatorname{om}_{S}\left(\mathscr{F}, \mathbf{D}_{S} \mathbf{D}_{S} \mathscr{G}\right) \\
& \cong \mathbf{D}_{S}\left(\mathscr{F} \otimes_{\Lambda}^{\mathbf{L}} \mathbf{D}_{S} \mathscr{G}\right),
\end{aligned}
$$

where the first comes from the reflexivity of $\mathscr{G}$ proved in iii., and the second is an easy exercise in tensor-hom adjunction. The result now follows from parts ii. and iii.

For part v., the habitual isomorphism $R f^{!} \mathbf{D}_{S} \cong \mathbf{D}_{T} f^{*}$ together with the reflexivity of $\mathscr{F}$ give isomorphisms

$$
R f^{!} \mathscr{F} \cong R f^{!} \mathbf{D}_{S} \mathbf{D}_{S} \mathscr{F} \cong \mathbf{D}_{T} f^{*} \mathbf{D}_{S} \mathscr{F},
$$

so the result now follows from part iii.

\section{References}

[AF59] Aldo Andreotti and Theodore Frankel, The Lefschetz theorem on hyperplane sections, Ann. of Math. (2) 69 (1959), 713-717. MR 0177422

[Bar76] Wolfgang Bartenwerfer, Der erste Riemannsche Hebbarkeitssatz im nichtarchimedischen Fall, J. Reine Angew. Math. 286/287 (1976), 144-163. MR 0422680

[Ber93] Vladimir G. Berkovich, étale cohomology for non-Archimedean analytic spaces, Inst. Hautes Études Sci. Publ. Math. (1993), no. 78, 5-161 (1994). MR 1259429

[Ber94] _ _ Vanishing cycles for formal schemes, Invent. Math. 115 (1994), no. 3, 539-571. MR 1262943

[Ber96] _ _ Vanishing cycles for formal schemes. II, Invent. Math. 125 (1996), no. 2, 367-390. MR 1395723

[Ber15] _ Finiteness theorems for vanishing cycles of formal schemes, Israel J. Math. 210 (2015), no. 1, 147-191. MR 3430272

[BGR84] S. Bosch, U. Güntzer, and R. Remmert, Non-Archimedean analysis, Grundlehren der Mathematischen Wissenschaften [Fundamental Principles of Mathematical Sciences], vol. 261, Springer-Verlag, Berlin, 1984, A systematic approach to rigid analytic geometry. MR 746961

[Con99] Brian Conrad, Irreducible components of rigid spaces, Ann. Inst. Fourier (Grenoble) 49 (1999), no. 2, 473-541. MR 1697371

[DG94] G. Dethloff and H. Grauert, Seminormal complex spaces, Several complex variables, VII, Encyclopaedia Math. Sci., vol. 74, Springer, Berlin, 1994, pp. 183-220. MR 1326621

[Elk73] Renée Elkik, Solutions d'équations à coefficients dans un anneau hensélien, Ann. Sci. École Norm. Sup. (4) 6 (1973), 553-603 (1974). MR 0345966 
[Gre76] Silvio Greco, Two theorems on excellent rings, Nagoya Math. J. 60 (1976), 139-149. MR 0409452

[Han17] David Hansen, A primer on reflexive sheaves, Appendix to the preprint "On the Kottwitz conjecture for local Shimura varieties" by Tasho Kaletha and Jared Weinstein.

[Hub96] Roland Huber, Étale cohomology of rigid analytic varieties and adic spaces, Aspects of Mathematics, E30, Friedr. Vieweg \& Sohn, Braunschweig, 1996. MR 1734903

[ILO14] Luc Illusie, Yves Laszlo, and Fabrice Orgogozo, Travaux de Gabber sur l'uniformisation locale et la cohomologie étale des schémas quasi-excellents, Astérisque 363-364 (2014).

[Kie67a] Reinhardt Kiehl, Der Endlichkeitssatz für eigentliche Abbildungen in der nichtarchimedischen Funktionentheorie, Invent. Math. 2 (1967), 191-214. MR 0210948

[Kie67b] _ Die de Rham Kohomologie algebraischer Mannigfaltigkeiten über einem bewerteten Körper, Inst. Hautes Études Sci. Publ. Math. (1967), no. 33, 5-20. MR 0229644

[Lüt93] W. Lütkebohmert, Riemann's existence problem for a p-adic field, Invent. Math. 111 (1993), no. 2, 309-330. MR 1198812

[Mit] Kentaro Mitsui, Criterion for minimality of rigid analytic surfaces, preprint, https://www.math.kyoto-u.ac.jp/preprint/2009/20mitsui.pdf.

[Nor02] Madhav V. Nori, Constructible sheaves, Algebra, arithmetic and geometry, Part I, II (Mumbai, 2000), Tata Inst. Fund. Res. Stud. Math., vol. 16, Tata Inst. Fund. Res., Bombay, 2002, pp. 471-491. MR 1940678

[SGA73] Théorie des topos et cohomologie étale des schémas. Tome 3, Lecture Notes in Mathematics, Vol. 305, Springer-Verlag, Berlin-New York, 1973, Séminaire de Géométrie Algébrique du Bois-Marie 1963-1964 (SGA 4), Dirigé par M. Artin, A. Grothendieck et J. L. Verdier. Avec la collaboration de P. Deligne et B. Saint-Donat. MR 0354654

[Sta17] The Stacks Project Authors, Stacks Project, http://stacks.math.columbia.edu, 2017.

[Tem17] Michael Temkin, Functorial desingularization over $\mathbf{Q}$ : boundaries and the embedded case, preprint, to appear in Israel J. Math.

[Val75] Paolo Valabrega, On the excellent property for power series rings over polynomial rings, J. Math. Kyoto Univ. 15 (1975), no. 2, 387-395. MR 0376677

[Val76] _ A few theorems on completion of excellent rings, Nagoya Math. J. 61 (1976), 127133. MR 0407007 